Research Article

\title{
YNCRG Inhibited Metabolic Syndrome Through Appetite Suppression and Improved Lipid Metabolism in Metabolic Syndrome Model Rats
}

Maya Kudo ${ }^{1}$, Misa Hayashi ${ }^{1}$, Peng Tian ${ }^{3}$, Danyang Liu ${ }^{4}$, Lili $\mathrm{Wu}^{3}$, Wei Li ${ }^{4}$, Zhaoyang Hong ${ }^{5}$, Yitang Zhao ${ }^{5}$, Toshiaki Nishigaki ${ }^{6}$, Masaaki Nishihara ${ }^{7}$, Kazuo Koike ${ }^{4}$, Tonghua Liu ${ }^{3}$, Ming Gao ${ }^{1,2}$, *

1. School of Pharmaceutical Science, Mukogawa Women's University, 11-68 Koshien Kyuban-cho, Nishinomiya, Hyogo 663-8179, Japan; E-Mails: 1422006@mwu.jp; hayasi27@mukogawa-u.ac.jp; gaoming@mukogawa-u.ac.jp

2. Institute for Biosciences, Mukogawa Women's University, 11-68 Koshien Kyuban-cho, Nishinomiya, Hyogo 663-8179, Japan

3. Key Laboratory of Health Cultivation of the Ministry of Education, Beijing University of Chinese Medicine, 11 North 3rd-ring East Road, 100029 Chaoyang District, Beijing, China; E-Mails: delladepp@yeah.net; gingnido_566@163.com; thliu@vip.163.com

4. Faculty of Pharmaceutical Sciences, Toho University, 2-2-1 Miyama, Funabashi, Chiba 274-8510, Japan; E-Mails: 3020008r@st.toho-u.jp; liwei@phar.toho-u.ac.jp; koike@phar.toho-u.ac.jp

5. Hundred herbs source science and technology group Co., Ltd, 101100 Room 204-1-143, Rigria House, Cuiping Tongzhou District, Beijing, China; E-Mails: yitong@hongrun-group.com; hongchaoyang@hongrun-group.com

6. M\&K Laboratories Co., Ltd, 1286-18 Azusagawayamato, Matsumoto, Nagano 390-1701, Japan; E-Mails:mk-info@thisismk.co.jp

7. Protein Chemical Co., LTD, 1-1-7 Uchisaiwai-cho, Chiyoda-ku, Tokyo 100-0011, Japan; E-Mails: nishihara@prochemi.co.jp

* Correspondence: Ming Gao; E-Mail: gaoming@mukogawa-u.ac.jp

Academic Editor: Rafat A. Siddiqui

Special Issue: Managing Obesity through Nutraceuticals

OBM Integrative and Complementary Medicine 2020 , volume 5 , issue 3

doi:10.21926/obm.icm.2003033
Received: April 13, 2020

Accepted: June 30, 2020

Published: July 09, 2020

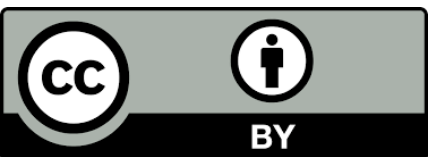

(C) 2020 by the author. This is an open access article distributed under the conditions of the Creative Commons by Attribution License, which permits unrestricted use, distribution, and reproduction in any medium or format, provided the original work is correctly cited. 


\begin{abstract}
Increased body fat mass can lead to lifestyle diseases such as hypertension, diabetes, and hyperlipidemia. Metabolic syndrome is a cluster of conditions that increases the onset of multiple diseases, such as cardiovascular diseases, stroke, and type 2 diabetes, which are closely related to lipid metabolism; therefore, new prevention approaches and treatments are needed. Our study investigated the effects and mechanism of YNCRG on anti-obesity in metabolic syndrome model SHR.Cg-Lepr ${ }^{\mathrm{cP}} / \mathrm{NDmcr}$ (SHRCP) rats. Eight-week-old male SHRCP rats were administered YNCRG or water by gavage for eight weeks and sacrificed. The tissues were immediately dissected for future experiments. We also used WKY rats as a rodent model. YNCRG induced reductions in food intake, body weight, and body fat mass in SHRCP rats. We also analyzed the mechanism of food intake regulation in the hypothalamus. Expression of POMC was significantly increased in the YNCRG group. However, JAK2 showed no change between the two groups. Phosphorylation levels of Akt (Ser473) and FoxO1 were significantly increased in the YNCRG group. Moreover, mTOR was significantly phosphorylated by YNCRG treatment. These results suggest that YNCRG can induce appetite suppression through the mTOR-Akt-FoxO1-POMC signaling pathway. YNCRG also induced a reduction in body fat mass. In adipose tissue, phosphorylation of AMPK was significantly increased in the YNCRG group. Also, LKB1 and PKA (upstream factors of AMPK), and ACC and HSL (downstream factors of AMPK) were significantly phosphorylated with the YNCRG treatment. Our results indicate that YNCRG can treat MetS by decreasing body fat mass of SHRCP rats through appetite suppression and improvement of lipid metabolism.
\end{abstract}

\title{
Keywords
}

YNCRG; metabolic syndrome; body fatty mass; appetite suppression; lipid metabolic pathway

\section{Introduction}

An increasing trend toward Western dietary patterns in Japan is considered to be a risk factor for obesity and insulin resistance, which are central to the development of the metabolic syndrome (MetS) [1, 2]. MetS is caused by an imbalance between energy intake and energy expenditure [3] and results in hypertension, high blood glucose, and diabetes. It is also a risk factor for arteriosclerosis. MetS is caused by overeating, stress, lack of exercise, and weight gain. Although drugs are administered when symptoms appear, there is concern that long-term drug use may adversely affect the human body. Therefore, the development of herbal medicine is desirable.

The molecular mechanism of MetS is still under investigation, with the involvement of multiple complicated signaling pathways. Recently, proopiomelanocortin (POMC), a food intake regulator protein, has been shown to affect appetite suppression. Two signaling pathways regulate POMCleptin signaling and insulin signaling. Binding of leptin to its specific receptor activates Janus kinase 2 (JAK2) and the signal transducer and activator of transcription 3 (STAT3) [4] and stimulates POMC expression in the hypothalamus. Insulin signaling begins with the binding of insulin to the 
insulin receptor (IR), resulting in phosphorylation of insulin receptor substrate 1 (IRS1) and protein kinase B (Akt). Akt is stimulated, and phosphorylation activity increases, leading to phosphorylation of Forkhead box $\mathrm{O} 1$ (FoxO1) by Akt $[5,6]$. Finally, phosphorylated FoxO1 increases the expression of POMC, causing appetite suppression.

Adipose tissue plays a major role in energy homeostasis, lipid metabolism, and insulin action [7]. AMP-activated protein kinase (AMPK) is a crucial cellular energy sensor [8]. Calciumcalmodulin/dependent protein kinase (CaMKK) and liver kinase B1 (LKB1) are the two major enzymes that catalyze AMPK phosphorylation [9, 10]. Furthermore, they can modulate the expression of downstream target genes involved in lipogenesis and lipolysis such as acetyl-CoA carboxylase (ACC), fatty acid synthase (FAS) and hormone-sensitive lipase (HSL) [11, 12]. AMPK is considered a potential target in the treatment of metabolic disorders.

For centuries, traditional herbal medicines have played an important role in maintaining good health. Generally, herbal medicines have been considered for the treatment of diseases with multiple conditions such as MetS. In this study, a formulation named YNCRG was selected to evaluate the anti-obesity and anti-MetS activities. The formulation contained Tochu leave extract as the main ingredient. The other ingredients were Red koji rice (Monascus purpureus), Noni (Morinda citrifolia), L-Citrulline (L-Cit), and gamma-aminobutyric acid (GABA). Previous studies have reported that Tochu can ameliorate MetS. In this study, we developed a new mixture of five natural resources named YNCRG, which has Tochu as the major ingredient. YNCRG has been shown to improve lipid metabolism, treat hypertension, and suppress appetite.

Tochu (Eucommia ulmoides Oliv.), belonging to the family of Eucommiaceae, has a long history of use in traditional Chinese medicine. Tochu leaves have been paid more attention due to its antiMetS activities, such as hypolipidemic [13], antibacterial [14], hepatoprotective [15], and antihypertensive [16] effects. Red koji rice, an edible fungus, has been widely used to produce wines and other fermented foods, particularly in Asia $[17,18]$. In a recent study, Red koji rice showed an anti-obesity effect in high-fat diet obese rats [19]. Noni is a traditional medicinal plant [20] with a long history of use as a food in tropical regions [21]. Noni has been reported to have a broad range of therapeutic effects and effective remedy for cold, influenza, diabetes, hypertension, cancer, and other illnesses [22, 23]. L-Citrulline (L-Cit) is a free amino acid found in cucurbits such as watermelon [24]. In our previous report, L-Cit was shown to have an anti-obesity effect due to appetite suppression [25]. GABA is one of the natural amino acids found in plants, animals, and humans. In particular, GABA $\mathrm{B}$ receptors regulate POMC expression [26].

SHR.Cg-Lepr ${ }^{\mathrm{cP}} / \mathrm{NDmcr}$ (SHRCP) rats spontaneously develop moderately elevated blood glucose with hyperphagia, insulin resistance, obesity, dyslipidemia, and hypertension, traits that resemble those of human pre-diabetes and MetS $[27,28]$. This study investigated the mechanism of action and possible synergistic effects of YNCRG on MetS in terms of appetite suppression and lipid metabolic pathways in SHRCP rats.

\section{Materials and Methods}

All procedures were carried out in accordance with the guiding principles for the care and use of animals in the field of physiological sciences established by the Physiological Society of Japan. The study was approved by the Ethics Committee of Laboratory Animals at Mukogawa Women's University (grant number: P-06-2018-01-A; date of approval: November 27, 2018). 


\subsection{Preparation of YNCRG}

YNCRG contains Eucommia ulmoides (Tochu leaves was supplied by Maruzen Pharmaceuticals Co., Ltd, Hiroshima, Japan, Lot number: 01101047), Monascus purpureus (Red koji rice was supplied by Kobayashi Pharmaceutical Co., Ltd, Osaka, Japan, Lot number: 320-180801), Morinda citrifolia (Noni was supplied by Earth Ship Co., Ltd, Osaka, Japan, Lot number: 40004), L-Citrulline (L-Cit was supplied by Protein Chemical Co., LTD, Tokyo, Japan, Lot number: 75A05CT) and gamma-aminobutyric acid (GABA, which was supplied by Coach Boueki Co., Ltd, Osaka, Japan, Lot number: 20180104), in a proportion of 4:2:2:1:1.

Dosage: We determined the dose of YNCRG based on a human weighting $50 \mathrm{~kg}$ taking $9 \mathrm{~g}$ YNCRG daily. We used 15 times this amount in SHRCP rats and decided on an experimental period for which the effect of YNCRG on metabolic syndrome would be the strongest.

\subsection{Experimental Animals}

SHRCP rats and the Wister Kyoto (WKY) rats (Japan SLC, Inc., Shizuoka, Japan) were 6-week-old males. All rats were kept at $22-24{ }^{\circ} \mathrm{C}$ under artificial lighting with a $12 \mathrm{~h} /$ daylight and dark cycle. Rats were fed CE-2 (normal chow, CLEA Japan, Inc. Japan) for two weeks to stabilize their metabolic condition. Rats were then randomly assigned to three groups ( $n=9,8,8$ in each group): a control group (normal water); a YNCRG group (water with $3.6 \mathrm{~g} / \mathrm{kg}$ body weight/day YNCRG); and a WKY group (normal water). We administered water or YNCRG to SHRCP rats by gavage, measured body weight every day, and recorded food and water intake once a week. Blood pressure was measured once every two months, 24-h-urine was collected once a month, and an oral glucose tolerance test (OGTT) was performed after an 8-week treatment period.

All rats were sacrificed after the 8-week treatment period after $24 \mathrm{~h}$ of fasting. Rats were anesthetized with isoflurane and all efforts were made to minimize suffering. Blood samples were collected from the abdominal aorta using injection needles and sera were centrifuged, frozen, and stored at $-20{ }^{\circ} \mathrm{C}$ until later analyses. The tissues (liver, kidneys, heart, fat, aortic, adrenal gland, pancreases, brain) were immediately dissected and cleaned to measure organs and tissue weights, and organs and tissues were promptly frozen in liquid nitrogen and stored at $-80{ }^{\circ} \mathrm{C}$ for western blotting and real-time PCR analysis.

All procedures were carried out in accordance with the guiding principles for the care and use of animals in the field of physiological sciences established by the Physiological Society of Japan. The study was approved by the Ethics Committee of Laboratory Animals at Mukogawa Women's University (permit Number: P-06-2018-01-A).

\subsection{Biochemical Analysis of Serum and Urine}

Non-fasting blood glucose (NFBG) was measured using a self-testing glucose kit at the end of the 8-week treatment period. Serum total cholesterol (TC), triglycerides (TG), aspartate aminotransferase (AST), and alanine aminotransferase (ALT) were measured enzymatically using an assay kit (Wako, Japan). We asked a vendor to measure serum albumin (ALB), blood urea nitrogen (BUN), creatinine (Cre), uric acid (UA), urine-total protein (TP), urine-urine nitrogen (UN) and urine-Cre. 


\subsection{Blood Pressure and Heart Rate Measurements}

Systolic blood pressure (SBP), diastolic blood pressure (DBP), and heart rate were measured using a sphygmomanometer (UR-1000, Ueda Co., Chiba) with the tail-cuff method once every two weeks.

\subsection{OGTT}

Blood was sampled via the tail vein after 12-18 $\mathrm{h}$ of fasting. Fasting blood glucose (FBG) was quantified using the glucose oxidase method at $0,30,60,90,120 \mathrm{~min}$ after glucose $(1 \mathrm{~g} / \mathrm{kg})$ loading in the OGTT. The area under the curve (AUC) was then calculated from the serum glucose content [29].

\subsection{Assessment of Body Composition}

Body composition was analyzed by bioelectrical impedance analysis (BIA) (ImpediVET, ImprdiMed Ltd., Brisbane, Australia) as previously described [30].

\subsection{Primary and Secondary Antibodies}

Western blotting was performed with the following commercially available antibodies: antirabbit Akt, anti-rabbit phosphor-Akt (Ser473) anti-rabbit phosphor-Akt (Thr308), anti-rabbit FoxO1, anti-rabbit phosphor-FoxO1, anti-rabbit phosphor-IRS1 (Ser1101), anti-rabbit phosphormammalian target of rapamycin (mTOR) (Ser2448), anti-rabbit phosphor-S6 kinase (S6K) (Ser240/244), anti-rabbit phosphor-janus activating kinase 2 (JAK2) (Ser1007/1008), anti-rabbit AMPK, anti-rabbit phosphor-AMPK, anti-rabbit phosphor-CaMKK, anti-rabbit phosphor-LKB1, antirabbit phosphor-protein kinase $\mathrm{A}$ (PKA), anti-rabbit ACC, anti-rabbit phosphor-ACC, anti-rabbit HSL, anti-rabbit phosphor-HSL, anti-rabbit CCAAT/enhancer-binding protein (C/EBP) $\alpha$, anti-rabbit C/EBP $\beta$, anti-rabbit peroxisome proliferator-activated receptor $\gamma$ (PPAR $\gamma$ ), anti-rabbit IgG and antimouse IgG from Cell Signaling Technology (Beverly, MA, USA); anti-rabbit POMC, anti-goat agoutirelated protein (Agrp), anti-rabbit phospho-IRS1 (Tyr465), anti-rabbit IRS1 and ant-goat IgG from Santa Cruz Biotechnology; and anti-mouse $\beta$-Actin from Sigma (St. Louis, MO, USA).

\subsection{Protein Extraction Experiments}

Hypothalamus and fat tissues were homogenized in ice-cold homogenization buffer containing $50 \mathrm{mM}$ Tris- $\mathrm{HCl}$ (pH 7.4), $100 \mathrm{mM} \mathrm{NaCl}, 1 \%$ Nonident P-40, 0.25\% Na deoxycholate, $0.1 \%$ SDS, 1 mM EDTA, $50 \mathrm{mM} \mathrm{NaF}, 2 \mathrm{mM} \mathrm{Na}_{3} \mathrm{VO}_{4}, 30 \mathrm{mM}$ Na pyrophosphate, $2 \mathrm{mM}$ PMSF, $1 \mathrm{mM}$ benzanidine, $0.02 \mathrm{~g} / \mathrm{mL}$ trypsin inhibitor, $0.02 \mathrm{~g} / \mathrm{mL}$ leupeptin, and $0.02 \mathrm{~g} / \mathrm{mL}$ aprotinin. After placing in an ice bath for $30 \mathrm{~min}$, the samples were centrifuged at $12,000 \mathrm{rpm}$ for $10 \mathrm{~min}$. The supernatants were isolated to obtain protein samples. Proteins were extracted by boiling the tissue samples in 0.5 $\mathrm{mM}$ Tris- $\mathrm{HCl}$ (pH 6.8), glycerol, 10\% SDS, 0.1\% bromophenol blue, and 2-mercaptethanol [25]. 


\subsection{Western Blot Analysis}

Proteins (10-20 $\mathrm{\mu g} /$ Lane) were electrophoresed using 10-12.5\% SDS-PAGE gel at $100 \mathrm{~V}$ for $90-$ $120 \mathrm{~min}$. We used the same gel and a separate gel for western blot analysis to assess experimental progress. After fractionating, proteins were transferred onto a polyvinylidene difluoride (PVDF) membrane (Amersham Life Science Inc. Buckinghamshire) at $100 \mathrm{~mA}$ for 1-2 h. The membranes were blocked with Blocking One or Blocking One-P (Nacalai Tesque, Japan) for $30 \mathrm{~min}$. After appropriate blocking, the membranes were incubated overnight with the primary antibody in antibody solution 1 (Toyobo, Japan). They were then washed with TTBS containing $1 \mathrm{M} \mathrm{Tris-HCl}(\mathrm{pH}$ 7.5), $\mathrm{NaCl}$, and 20\% Tween 20 and finally incubated for $1 \mathrm{~h}$ with a 1:5000-2000 dilution of antirabbit, goat and mouse IgG-horseradish peroxidase. Detection was achieved using a Chemi-Lumi One Super (Nacalai Tesque, Japan). $\beta$-Actin was used as an internal control. Protein band densities were analyzed using Image J software from the National Institutes of Health [25].

\subsection{RNA Extraction and Real-Time PCR}

otal RNA was isolated using Sepasol (R)-RNA I Super G (Nacalai Tesque, Japan) from liver tissue and quantified using spectrophotometry at $260 \mathrm{~nm}, 280 \mathrm{~nm}$, and $320 \mathrm{~nm}$. RNA was reversetranscribed into cDNA using ReverTra Ace ${ }^{\circledR}$ qPCR RT Master Mix with a gDNA remover (TOYOBO, Japan) according to the manufacturer's protocol and used for the amplification of target genes in real-time PCR with THUNDERBIRD SYBR qPCR Mix (TOYOBO, Japan). The specific primers were obtained from Thermo Fisher Scientific (Waltham, MA, USA). The PCR amplification was performed in Thermal Cycler Dice (Takara Bio Inc. Japan) as follows: 1 cycle at $95^{\circ} \mathrm{C}$ for $30 \mathrm{~s}$, and 40 cycles at $95^{\circ} \mathrm{C}$ for $5 \mathrm{~s}$ and $60^{\circ} \mathrm{C}$ for $30 \mathrm{~s}$. The fold differences in mRNA expression levels were analyzed using the $2{ }^{\triangle \Delta C T}$ method. Relative mRNA expression levels were calculated as the ratio of each transcript relative to the housekeeping gene, glyceraldehyde-3-phosphate dehydrogenase (GAPDH). The primer sequences are listed in Table 1 [29].

Table 1 Specific primer sequences.

\begin{tabular}{lll}
\hline Genes & Forward & Reverse \\
\hline GAPDH & AGAACATCATCCCTGCATCCA & CCGTTCAGCTCTGGGATGAC \\
Sirt1 & TGAAGCTGTTCGTGGAGATATTTTT & CATGATGGCAAGTGGCTCAT \\
FAS & GGCATCATTGGGCACTCCTT & GCTGCAAGCACAGCCTCTCT \\
\hline
\end{tabular}

\subsection{LC-MS Analysis}

LC-ESI-MS analysis was carried out using an LCMS-8040 triple quadrupole LC/MS/MS mass spectrometer (Shimadzu Co., Ltd., Kyoto, Japan) with a YMC-Triart C18 column $(3.0 \mu \mathrm{m}, 150 \times 20$ $\mathrm{mm}$ I.D.). The flow rate was $0.2 \mathrm{~mL} / \mathrm{min}$, and the column temperature was maintained at $35{ }^{\circ} \mathrm{C}$. The mobile phase was composed of $A\left(0.1 \%\right.$ formic acid in $\left.\mathrm{H}_{2} \mathrm{O}\right)$ and $B(0.1 \%$ formic acid in acetonitrile) with a gradient as follows: $10 \%$ B in $0-3 \mathrm{~min}, 10-50 \%$ B in $3-10 \mathrm{~min}, 50-90 \%$ B in $10-$ $40 \mathrm{~min}, 100 \% \mathrm{~B}$ in $40-50 \mathrm{~min}$. The mass spectrometer was operated in both positive- and negative ion modes with the full scan mode in the range of $100-1600 \mathrm{~m} / \mathrm{z}$. The optimized instrument 
settings were as follows: interface voltage, $4.5 \mathrm{kV}$ in the positive-ion mode, and $-3.5 \mathrm{kV}$ in the negative ion mode; collision voltage, $15 \mathrm{~V}$; dry gas, $15 \mathrm{~L} / \mathrm{min}$, and dry temperature $350{ }^{\circ} \mathrm{C}$.

YNCRG $(0.5 \mathrm{~g})$ was ultrasonically extracted with $\mathrm{MeOH}(20 \mathrm{~mL})$ at room temperature for $1 \mathrm{~h}$. The extracted solutions $(1 \mathrm{~mL})$ were purified using a Sep-Pak C18 plus short cartridge and diluted to $10 \mathrm{~mL}$. The solutions were filtered through a $0.45 \mu \mathrm{m}$ syringe filter. A volume of $2 \mu \mathrm{L}$ was injected for LC-ESI-MS analysis [31].

\subsection{Statistical Analysis}

Data are expressed as the mean \pm S.E.M. Statistical analyses of the data were performed using Student's t-test between the control group and the YNCRG group. A $p$-value of less than 0.05 was considered significant.

\section{Results}

\subsection{Effects of YNCRG on Body Weight, Food Intake, Organ and Tissue Weights, and Body Fat Mass in SHRCP and WKY Rats}

First, we examined the effects of YNCRG on body weight and food intake. When rats were treated daily with YNCRG or water for eight weeks, body weight, body weight gain and food intake were significantly lower in the YNCRG group compared with the control group (Fig. 1A, B, C). After sacrificing the rats, we measured organ and tissue weights and body fat mass. There were no significant differences in organ and tissue weights between the two groups (Table 2). However, body fat mass was significantly decreased in the YNCRG group (Fig. 1D).

Table 2 Effect of YNCRG on organ and tissue weights in SHRCP and WKY rats.

\begin{tabular}{llll}
\hline & Control & YNCRG & WKY \\
\hline Liver (mg/g (B.W.)) & $42.6 \pm 1.38$ & $38.0 \pm 2.68$ & $26.1 \pm 0.31$ \\
Kidneys (mg/g (B.W.)) & $2.89 \pm 0.16$ & $2.91 \pm 0.18$ & $3.57 \pm 0.41$ \\
$\begin{array}{l}\text { Adrenal gland (mg/g } \\
\text { (B.W.)) }\end{array}$ & $0.07 \pm 0.009$ & $0.08 \pm 0.004$ & $0.10 \pm 0.009$ \\
$\begin{array}{l}\text { Heart (mg/g (B.W.)) } \\
\text { Epididymal fat (mg/g }\end{array}$ & $20.1 \pm 0.73 \pm 0.15$ & $2.86 \pm 0.07$ & $3.01 \pm 0.05$ \\
(B.W.)) & & $19.0 \pm 1.05$ & $15.9 \pm 0.54$ \\
& & & \\
Brain (mg/g (B.W.)) & $2.83 \pm 0.04$ & $3.23 \pm 0.18^{* *}$ & $4.22 \pm 0.04$ \\
\hline
\end{tabular}

Eight-week-old male SHRCP and WKY rats were treated daily with YNCRG or water for eight weeks. Data are expressed as the mean \pm S.E.M.; $n=9,8,8$ in control, YNCRG, and WKY groups, respectively, ${ }^{* *} \mathrm{P}<0.01$ vs. control group. 


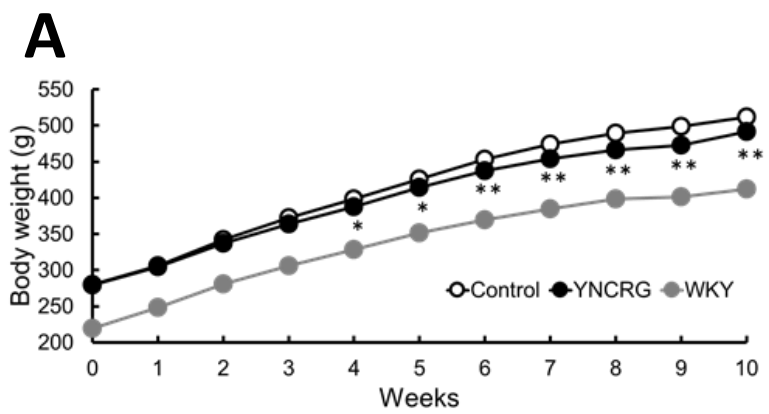

B

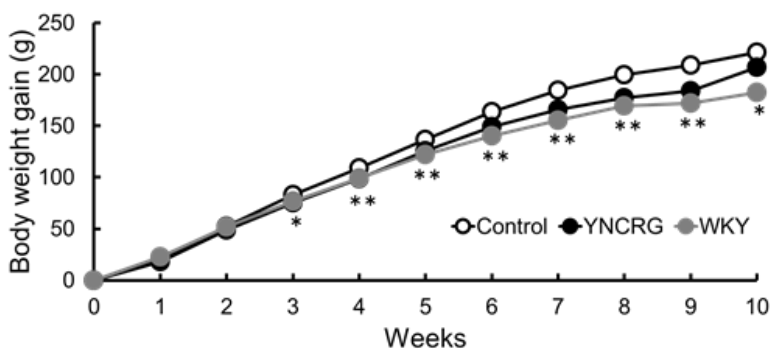

C

D
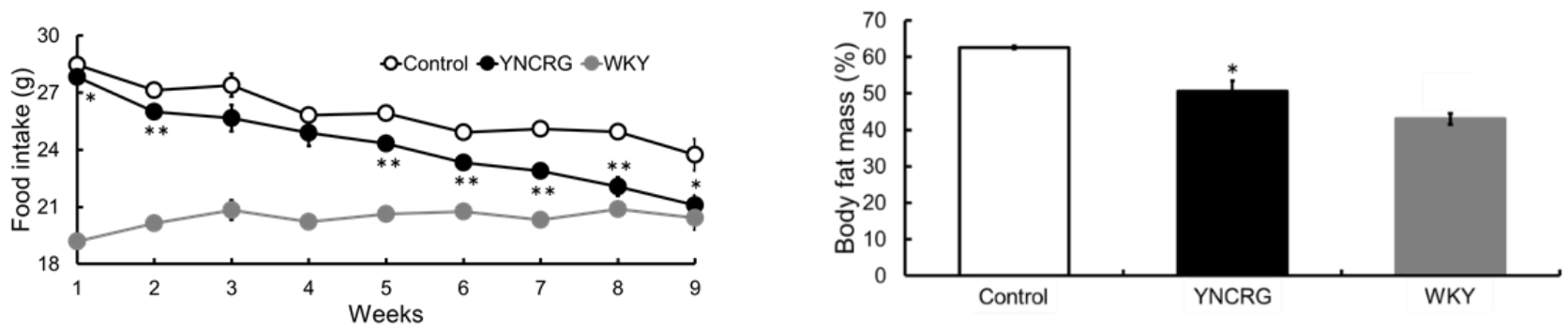

Figure 1 Effect of YNCRG on body weight, food intake, and organ and tissue weights in SHRCP and WKY rats.

Eight-week-old male SHRCP and WKY rats were treated daily with YNCRG or water for eight weeks. Bodyweight (A), body weight gain (B), food intake (C), and body fat mass (D). Data are expressed as the mean \pm S.E.M.; $n=9,8,8$ in control, YNCRG, and WKY groups, respectively, ${ }^{*} \mathrm{P}<0.05, * * \mathrm{P}<0.01$ vs. control group.

\subsection{Effects of YNCRG on Metabolic Parameter Levels in Serum in SHRCP and WKY Rats}

Next, we investigated various metabolic parameters in the serum in SHRCP and WKY rats. Serum TC tended to decrease after 8-week YNCRG treatment, but TG levels were unchanged between the two groups. After eight weeks of administration with YNCRG, the AST and ALT levels, which indicate liver function, were decreased in the YNCRG group suggesting that YNCRG does not have negative effects on the liver. Also, the non-fasting blood glucose level was significantly decreased in the YNCRG group. Further, we measured serum ALB, BUN, Cre, and UA, which are kidney damage markers. Their levels were not affected in the YNCRG group (Table 3 ).

Table 3 Effect of YNCRG on various serum blood parameters in SHRCP and WKY rats.

\begin{tabular}{llll}
\hline & Control & YNCRG & WKY \\
& & & \\
\hline TC $(\mathrm{mg} / \mathrm{dL})$ & $70.6 \pm 8.88$ & $67.0 \pm 10.2^{\#}$ & $54.5 \pm 10.7$ \\
$\mathrm{TG}(\mathrm{mg} / \mathrm{dL})$ & $612.1 \pm 40.3$ & $496.7 \pm 52.7$ & $21.0 \pm 4.47$ \\
AST $(\mathrm{IU} / \mathrm{L})$ & $140.4 \pm 24.1$ & $70.3 \pm 22.4^{*}$ & $85.3 \pm 21.0$ \\
ALT (IU/L) & $47.7 \pm 6.4$ & $35.1 \pm 10.4$ & $4.0 \pm 3.7$ \\
\hline
\end{tabular}




\begin{tabular}{llll}
\hline NFBG $(\mathrm{mg} / \mathrm{dL})$ & $143.1 \pm 12.0$ & $108.1 \pm 8.40 *$ & $96.1 \pm 3.41$ \\
ALB $(\mathrm{g} / \mathrm{dL})$ & $5.05 \pm 0.20$ & $6.88 \pm 0.01$ & $4.59 \pm 0.22$ \\
BUN (mg/dL) & $27.2 \pm 0.87$ & $29.8 \pm 2.01$ & $24.3 \pm 1.86$ \\
Cre (mg/dL) & $0.28 \pm 0.02$ & $0.33 \pm 0.03$ & $0.46 \pm 0.01$ \\
UA (mg/dL) & $2.49 \pm 0.34$ & $2.66 \pm 0.42$ & $1.56 \pm 0.20$ \\
\hline
\end{tabular}

Eight-week-old male SHRCP and WKY rats were treated daily with YNCRG or water for eight weeks. Data are expressed as the mean \pm S.E.M.; $n=9,8,8$ in control, YNCRG, and WKY groups, respectively, ${ }^{*} \mathrm{P}<0.05,{ }^{* *} \mathrm{P}<0.01$ vs. control group.

\subsection{Effects of YNCRG on Metabolic Parameter Levels in Urine in SHRCP and WKY Rats}

First, we measured the $24 \mathrm{~h}$ urine level after treatment for 4 and 8 weeks. The urine volume at eight weeks was significantly lower in the YNCRG group. However, urine TP, UN, and Cre were unchanged between the two groups (Table 4).

Table 4 Effects of YNCRG on urine level, urine TP, UN, and Cre in SHRCP and WKY rats.

\begin{tabular}{lllll}
\hline & Weeks & Control & YNCRG & WKY \\
& 4 & $27.3 \pm 1.76$ & $23.6 \pm 2.17$ & $14.9 \pm 1.25$ \\
Urine level (mL) & 8 & $26.4 \pm 1.25$ & $20.4 \pm 1.65^{*}$ & $16.4 \pm 0.46$ \\
& 4 & $24.4 \pm 3.57$ & $19.2 \pm 1.47$ & $15.7 \pm 1.92$ \\
& 8 & $42.0 \pm 5.90$ & $46.0 \pm 5.51$ & $9.04 \pm 0.84$ \\
Total protein (mg) & 8 & $567.3 \pm 27.9$ & $535.4 \pm 55.5$ & $327.9 \pm 36.2$ \\
& 8 & $385.5 \pm 13.4$ & $394.4 \pm 30.3$ & $187.5 \pm 20.1$ \\
Urine nitrogen & 4 & $8.26 \pm 0.47$ & $7.03 \pm 0.52$ & $8.46 \pm 0.79$ \\
(mg) & 4 & $6.28 \pm 0.39$ & $6.50 \pm 0.50$ & $7.01 \pm 0.48$ \\
& 8 & & & \\
\hline
\end{tabular}

Eight-week-old male SHRCP and WKY rats were treated daily with YNCRG or water for eight weeks. Data are expressed as the mean \pm S.E.M.; $n=9,8,8$ in control, YNCRG, and WKY groups, respectively, ${ }^{*} \mathrm{P}<0.05,{ }^{* *} \mathrm{P}<0.01$ vs. control group.

\subsection{Effects of YNCRG on SBP, DBP, and Heart Rate in Urine in SHRCP and WKY Rats}

SBP and DBP were significantly decreased in the YNCRG group compared with those in the control group. However, heart rate did not change between the two groups (Fig. 2A, B, C). 


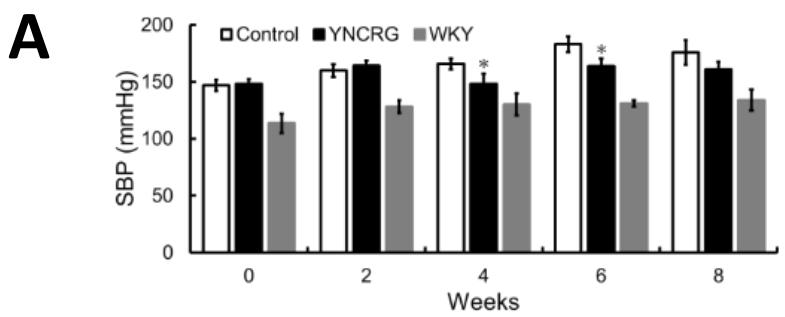

B
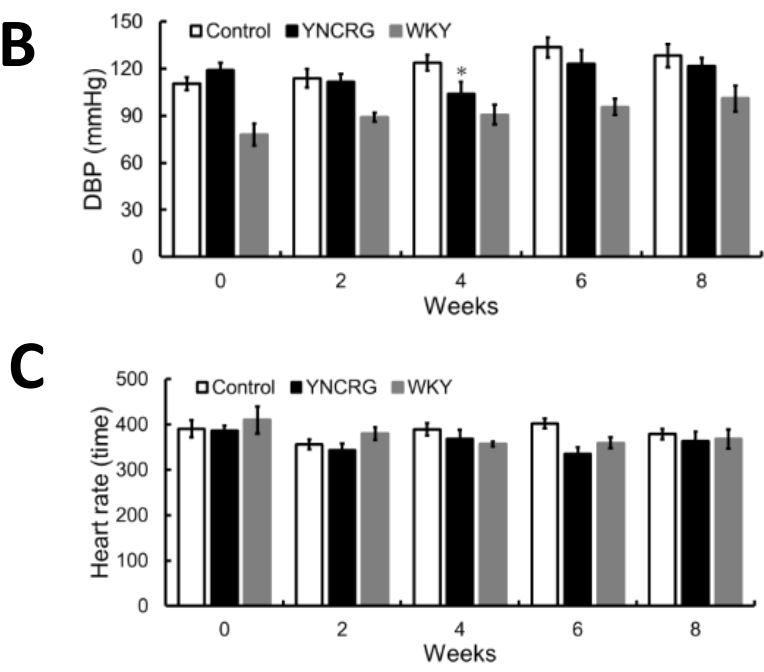

Figure 2 Effect of YNCRG on SBP, DBP and heart rate in SHRCP and WKY rats.

Eight-week-old male SHRCP and WKY rats were treated daily with YNCRG or water for eight weeks. SBP (A), DBP (B) and heart rate (C). Data are expressed as the mean \pm S.E.M.; $n=9,8,8$ in control, YNCRG and WKY groups, respectively, ${ }^{*} \mathrm{P}<0.05$ vs. control group.

\subsection{Effects of YNCRG on Glucose Tolerance in SHRCP and WKY Rats}

The OGTT showed that YNCRG inhibited blood glucose increase in a time-dependent manner and improved glucose metabolism (Fig. 3A, B).

A

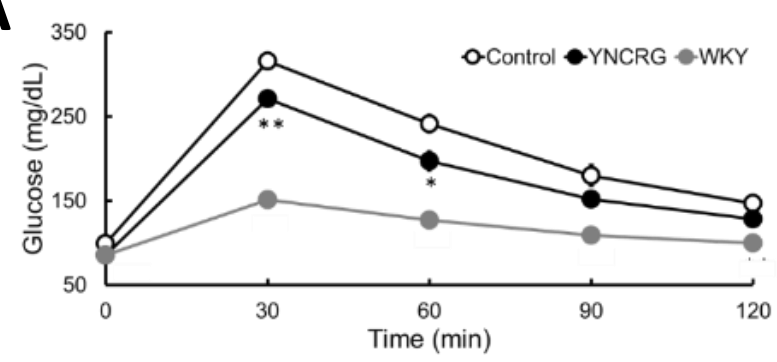

B

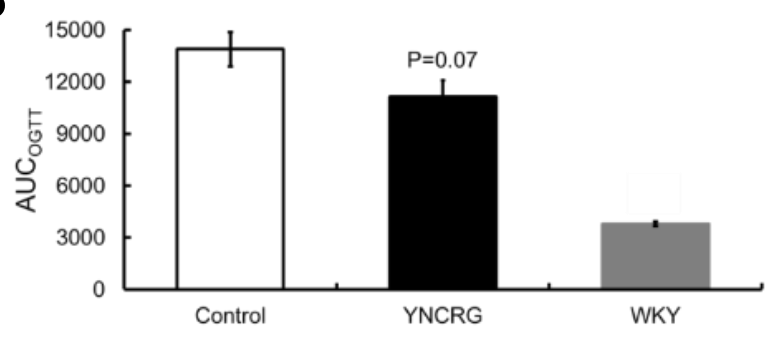

Figure 3 Effect of YNCRG on glucose tolerance in SHRCP and WKY rats.

Eight-week-old male SHRCP and WKY rats were treated daily with YNCRG or water for eight weeks. Blood glucose (A) and AUC (B). Data are expressed as the mean \pm S.E.M.; $n$ $=9,8,8$ in control, YNCRG and WKY groups, respectively, ${ }^{*} \mathrm{P}<0.05,{ }^{* *} \mathrm{P}<0.01$ vs. control group. 


\subsection{Effects of YNCRG on Food Intake Regulation Factors and Leptin Signaling in the Hypothalamus of SHRCP and WKY Rats}

As YNCRG affected food intake in SHRCP rats, we examined hypothalamic factors involved in food intake regulation. We measured the expression of Agrp, a peptide that promotes appetite, using Western blot analysis. The expression of Agrp was not affected by the YNCRG treatment (Fig. $4 A)$. On the other hand, expression of POMC, which suppresses appetite, was significantly higher in the YNCRG group than that in the control group, suggesting that YNCRG affects appetite suppression due to increased POMC expression (Fig. 4B). Two signaling pathways regulate POMC expression in the hypothalamus: leptin signaling and insulin signaling. First, to examine the effects of YNCRG on leptin signaling in the hypothalamus of SHRCP rats, we calculated the JAK2 phosphorylation ratio. The JAK2 phosphorylation ratio was not significantly different between the two groups, suggesting that leptin signaling was not stimulated by the YNCRG treatment (Fig. 4C).

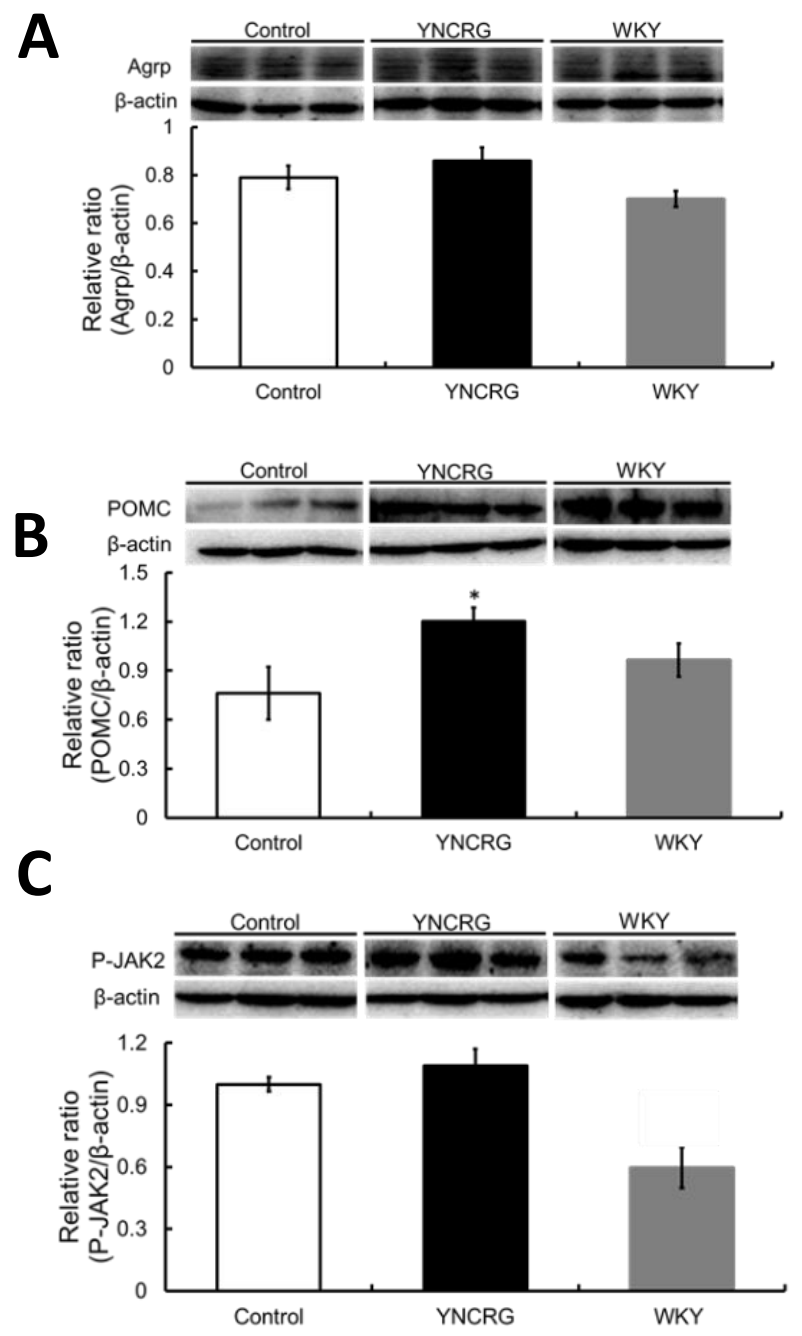

Figure 4 Effect of YNCRG on food intake regulation factors and leptin signaling in SHRCP and WKY rats.

Eight-week-old male SHRCP and WKY rats were treated daily with YNCRG or water for eight weeks. Agrp (A), POMC (B) and JAK2 (C). Data are expressed as the mean \pm S.E.M.; $\mathrm{n}=9,8,8$ in control, YNCRG and WKY groups, respectively, ${ }^{*} \mathrm{P}<0.05$ vs. control group. 


\subsection{Effects of YNCRG on insulin signaling in the hypothalamus of SHRCP and WKY rats}

As YNCRG was not involved in leptin signaling, we examined the effects of YNCRG on insulin signaling, which regulates POMC expression as well as leptin signaling, in SHRCP rats. We measured phosphorylated Akt expression as it is a central enzyme in insulin signaling. Akt (Ser473) phosphorylation significantly increased in the YNCRG group compared with the control group (Fig. $5 A$ ). On the other hand, the expression of phosphorylated Akt ( Thr 308) did not change in the two groups (Fig. 5B). Also, FoxO1, a downstream factor of Akt, was significantly phosphorylated in the YNCRG group (Fig. 5C). However, the expression of phosphorylated IRS1 (Ser1101, Tyr465) expressions was not significantly different between the two groups (Fig. 5D, E), suggesting that Akt did not phosphorylate via IRS1 signaling; therefore, we hypothesized that another pathway was stimulated by Akt. We measured phosphorylated mTOR expression as another target of Akt phosphorylation signaling. Expression of phosphorylated mTOR was significantly higher in the YNCRG group than in the control group (Fig. 5F), suggesting that YNCRG enhanced Akt phosphorylation by stimulating mTOR. However, S6K phosphorylation did not change with YNCRG treatment in SHRCP rats (Fig. 5G).
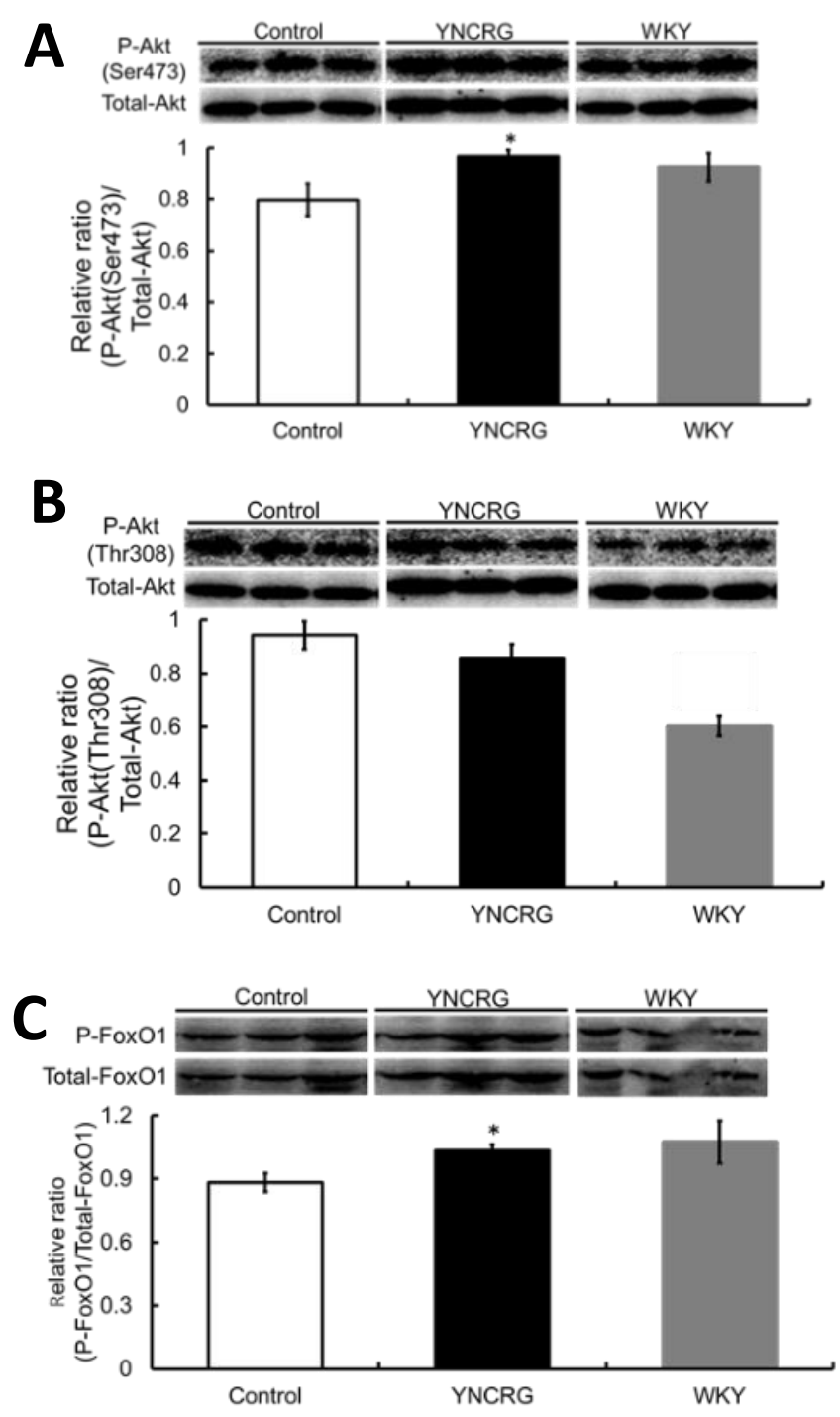

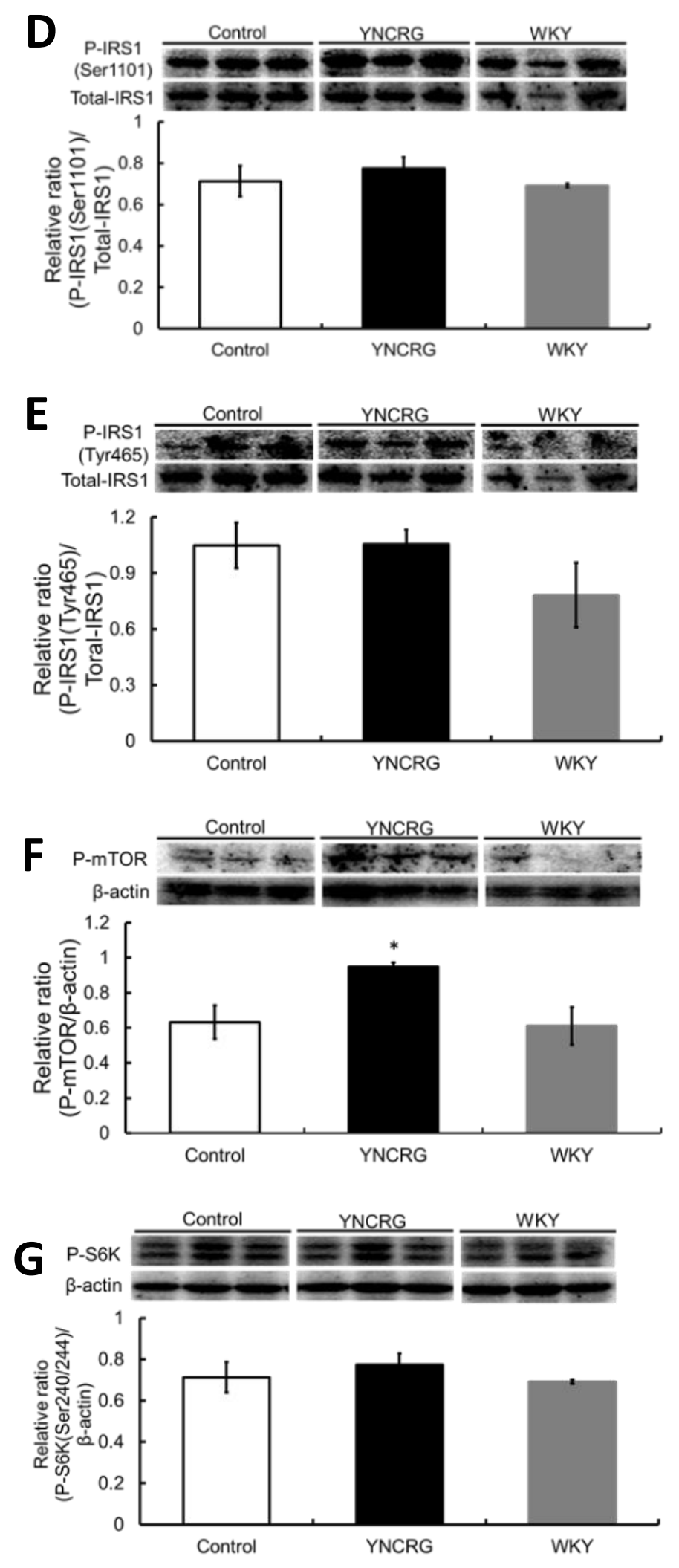

Figure 5 Effect of YNCRG on insulin signaling in the hypothalamus in SHRCP and WKY rats.

Eight-week-old male SHRCP and WKY rats were treated daily with YNCRG or water for eight weeks. Akt (Ser473, Thr308), FoxO1, IRS1 (Ser1101, Tyr465), mTOR and S6K (A-G). Data are expressed as the mean \pm S.E.M.; $n=9,8,8$ in control, YNCRG and WKY groups, respectively, $* P<0.05$ vs. control group. 


\subsection{Effects of YNCRG on the Expression of Lipid Metabolism-Related Proteins and Genes in the Epididymal Fat in SHRCP and WKY Rats}

As YNCRG affected body weight and body fat mass in SHRCP rats, we examined phosphorylation of the AMPK level, which is the main enzyme in the lipid metabolic pathway, using epididymal fat. AMPK phosphorylation of epididymal fat was significantly increased in the YNCRG group (Fig. 6A). Next, we measured phosphorylated LKB1 and PKA as they are upstream factors of AMPK. LKB1 and PKA phosphorylation significantly increased in the YNCRG group compared with that in the control group (Fig. 6B, C). However, phosphorylated CaMKK, Akt (Ser473), and the mRNA levels of Sirt1 were not significantly different between the two groups (Fig. 6D, E, F).

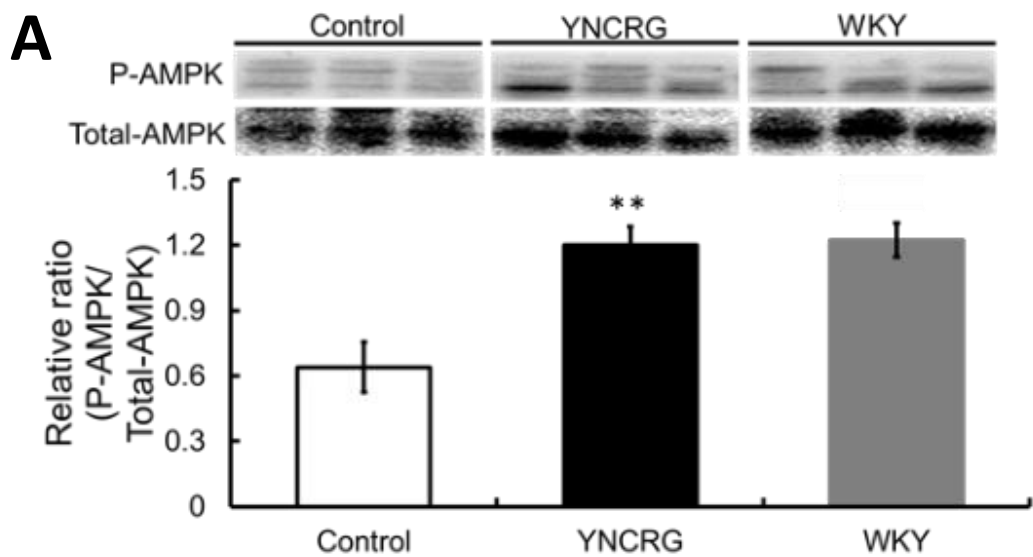

B
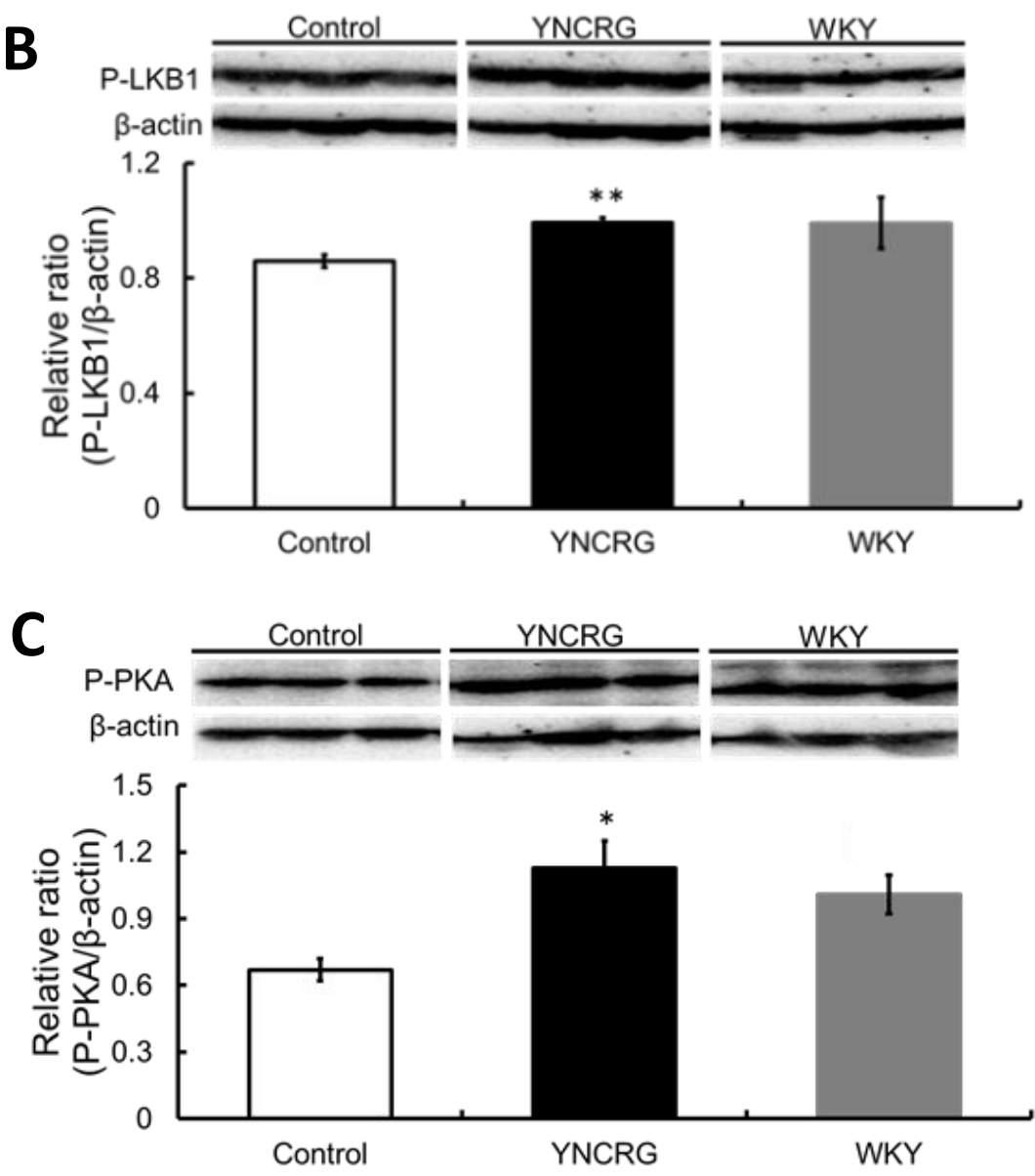


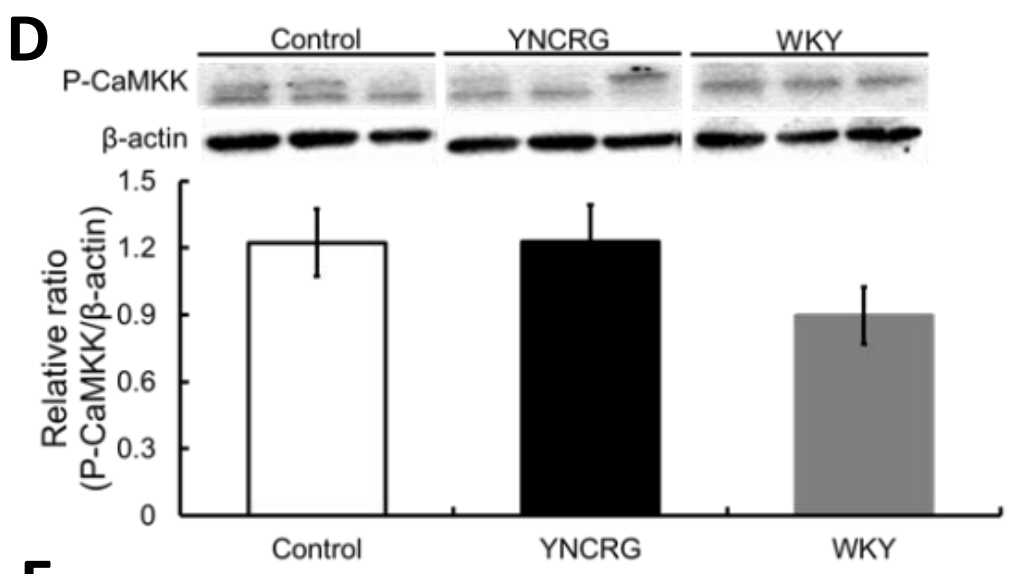

E
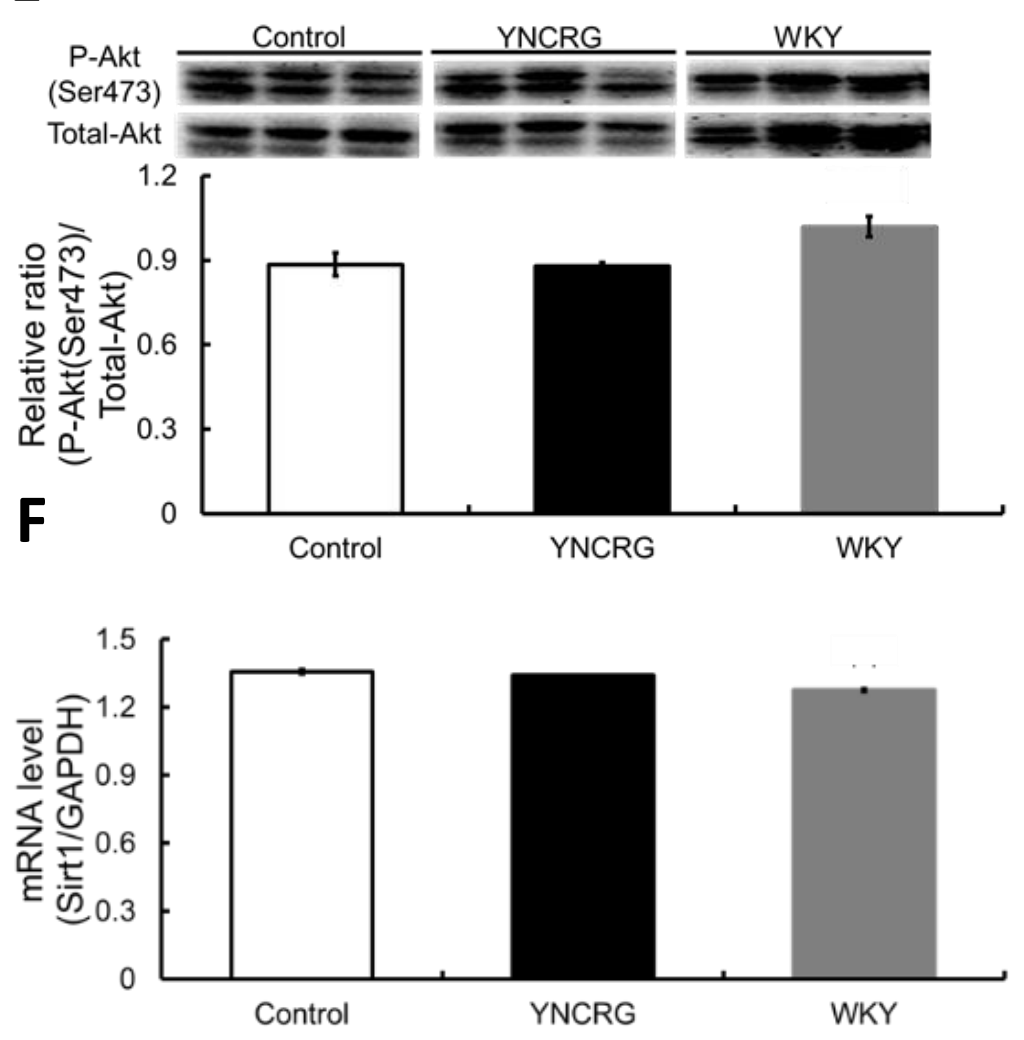

Figure 6 Effect of YNCRG on the expression of lipid metabolism-related proteins and genes in the epididymal fat in SHRCP and WKY rats.

Eight-week-old male SHRCP and WKY rats were treated daily with YNCRG or water for eight weeks. AMPK, LKB1, PKA, CaMKK, Akt (Ser473) and Sirt1 (A-F). Data are expressed as the mean \pm S.E.M.; $n=9,8,8$ in control, YNCRG and WKY groups, respectively, ${ }^{*} \mathrm{P}<0.05,{ }^{*} \mathrm{P}<0.01$ vs. control group.

\subsection{Effects of YNCRG on the Expression of AMPK Downstream Factors of Lipid Metabolism- Related Proteins and Genes in the Epididymal Fat in SHRCP and WKY Rats}

We also investigated downstream factors of AMPK, i.e., ACC, FAS, HSL, C/EBP $\alpha, C / E B P \beta$ and PPAR $\gamma$. The mRNA level of FAS and protein levels of C/EBP $\alpha, C / E B P \beta$, and PPAR $\gamma$ did not change in the two groups (Fig. 7A, B, C, D). However, the phosphorylation levels of ACC and HSL were significantly increased in the YNCRG group (Fig. 7E, F). 
A

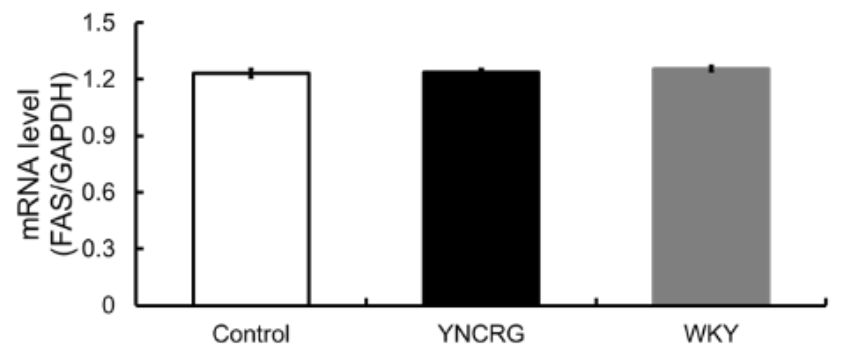

B

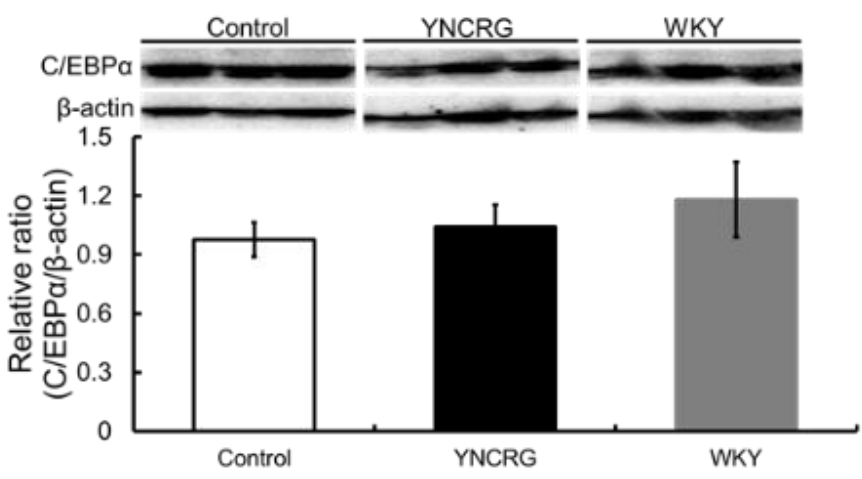

C

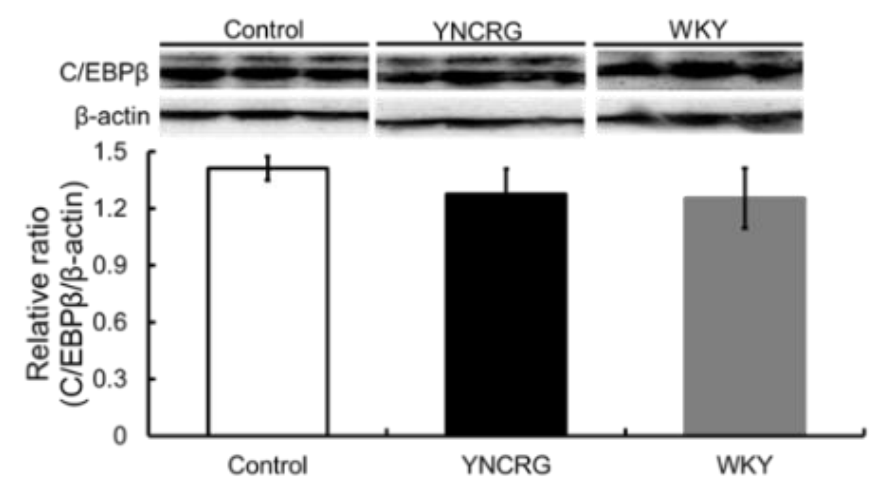

D

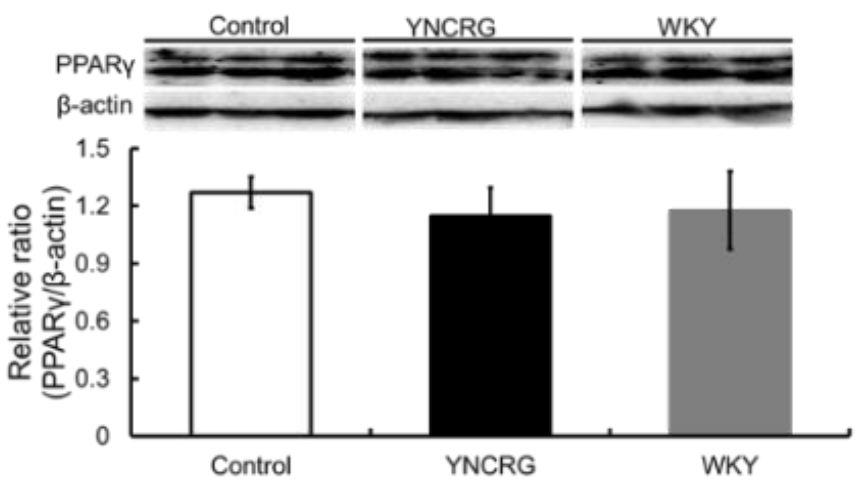

E
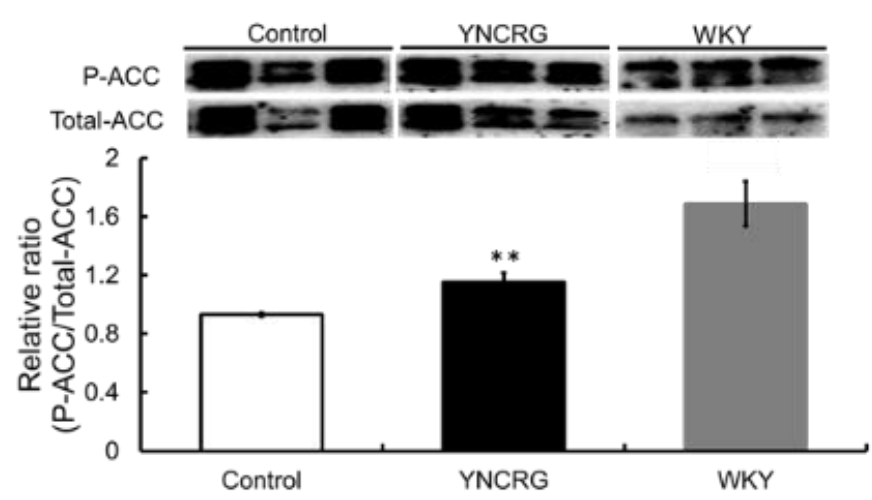


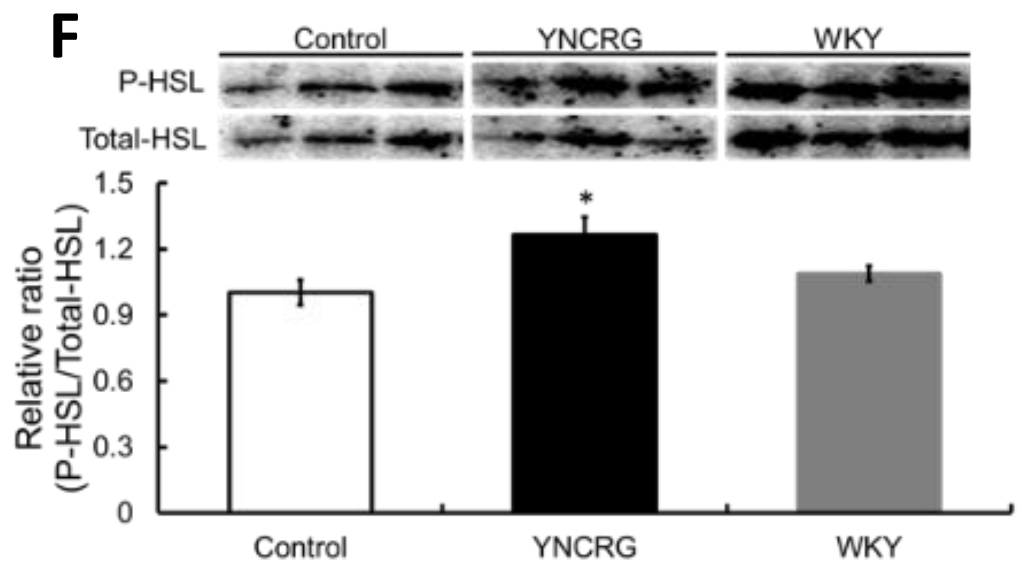

Figure 7 Effects of YNCRG on the expression of AMPK downstream factors of lipid metabolism-related proteins and genes in the epididymal fat in SHRCP and WKY rats.

Eight-week-old male SHRCP and WKY rats were treated daily with YNCRG or water for eight weeks. FAS, C/EBP $\alpha, C / E B P \beta, P P A R \gamma, A C C$ and HSL (A-F). Data are expressed as the mean \pm S.E.M.; $n=9,8,8$ in control, YNCRG, and WKY groups, respectively, ${ }^{*} P<0.05$, $* * P<0.01$ vs. control group.

\subsection{Identification of Main Constituents in YNCRG by LC-ESI-MS Analysis}

The chemical constituents in YNCRG were analyzed using LC-ESI method. The total ion chromatogram (TIC) of the sample solution in positive ion mode is shown in Figure 8. Based on the interpretation of the MS fragmentation patterns and also a comparison of those with available standard compounds, seven peaks were tentatively identified in the LC-MS chromatogram (Fig. 9). Peak 1 (chlorogenic acid), peak 5 (scandoside methyl ester), and peak $6((+)$-pinoresinol) were considered originally from Tochu leaves or Noni, and peak 2 (genistin acetate) was from red koji rice, while peaks 3 (mangiferin), peak 4 (dehydroconiferyl alcohol) and peak 7 (6ahydroxyadoxoside) were considered as constituents from Noni.

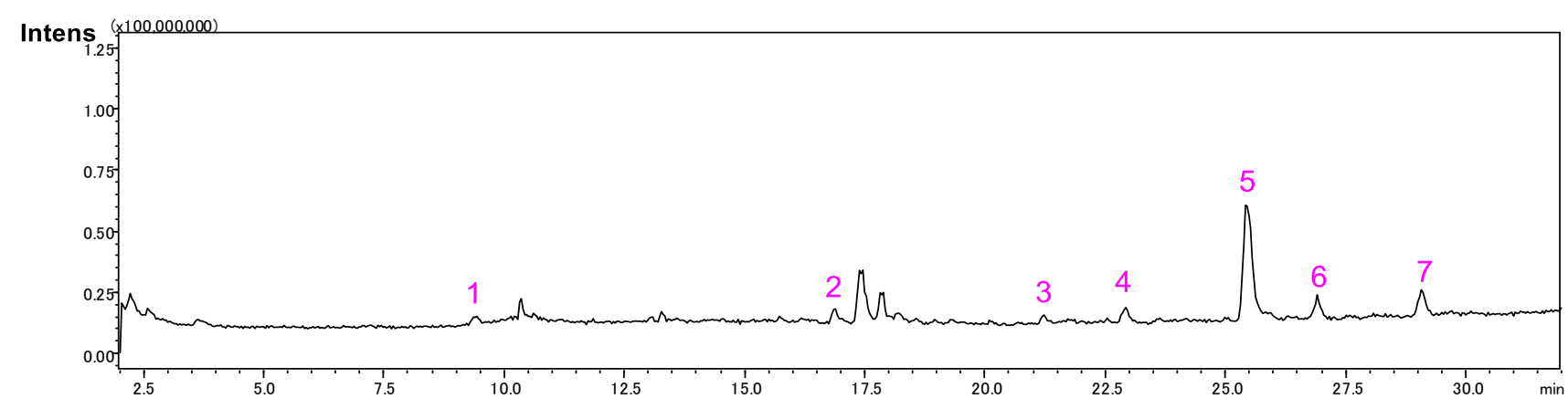

Figure 8 Total ion chromatogram of YNCRG by LC-ESI-MS in positive ion mode. Note. Two peaks at 17.5 min were from the background and not identified. 

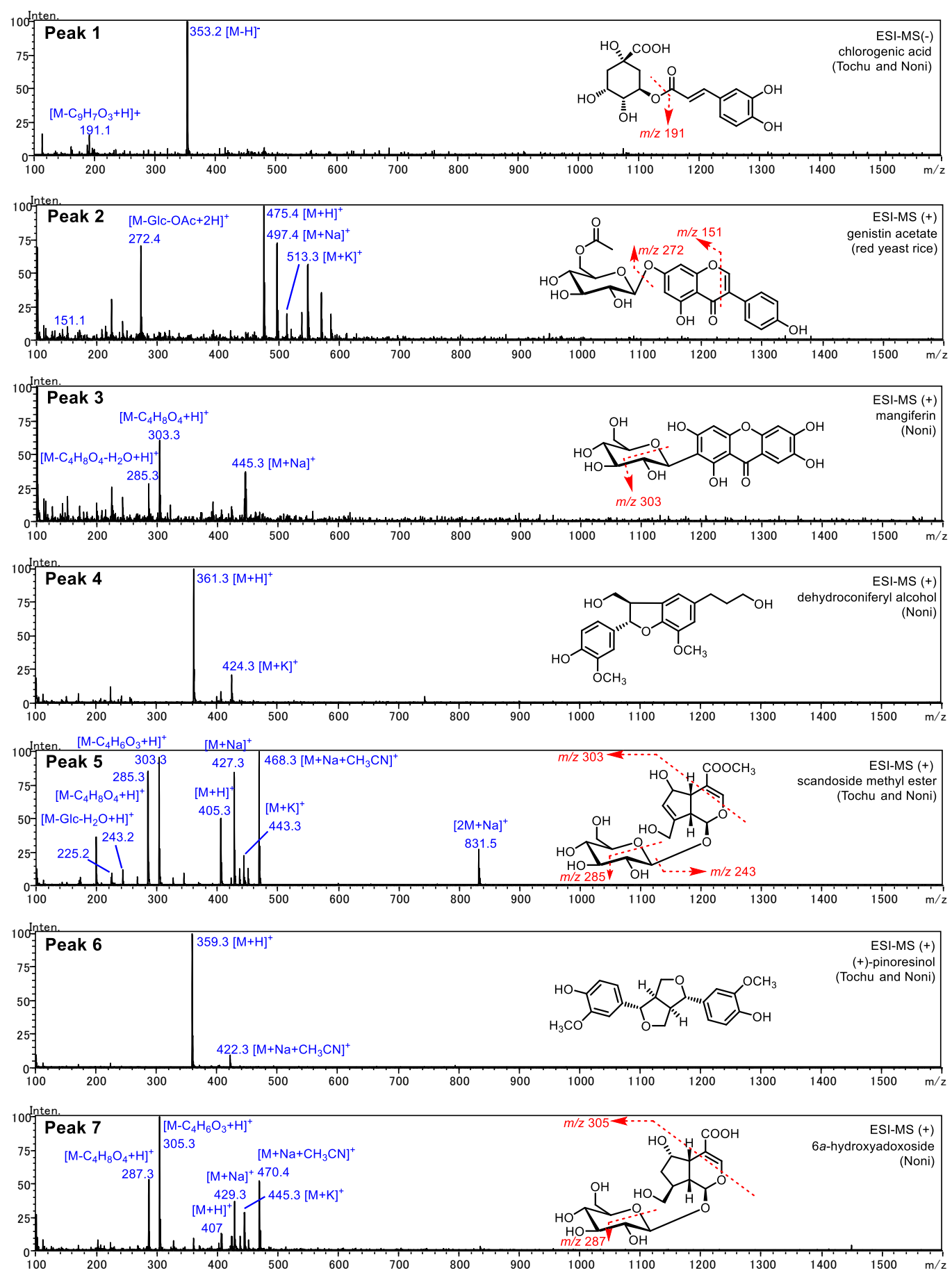

Figure 9 LC-ESI-MS spectra and proposed fragmentation pathways of peak 1-7.

(-) ESI-MS spectrum of peak 1 and (+) ESI-MS spectra of peaks 2-7.

\section{Discussion}

The main result of this study was that YNCRG inhibited body weight gain by the reduction in body fat mass via appetite suppression and an improvement in the lipid metabolic pathway in SHRCP rats. The underlying mechanisms were identified as increased expression of POMC in the hypothalamus, inhibition of lipogenesis, and promotion of lipolysis in the adipose tissue. 
Two signaling pathways, leptin signaling, and insulin signaling, regulate POMC expression. Leptin is a key regulator of feeding and long-term energy homeostasis that acts on discrete neuronal pathways to reduce food intake and body fat content [32]. One mechanism involved in leptin regulation of cellular function is the activation of JAK2 and STAT3 signaling [33, 34], and this appears to play a major role in the energy homeostasis mechanism. In this study, we showed that YNCRG does not phosphorylate JAK2, which is involved in leptin signaling. These results suggest that the change in POMC neuronal expression is not related to leptin signaling in YNCRG treatment.

Akt is the central enzyme in insulin signaling, and Akt activity is regulated by various factors such as IR-IRS1 signaling and mTOR signaling. We clarified that YNCRG increased the ratio of phosphorylated Akt (Ser473) in the hypothalamus of SHRCP rats. YNCRG did not affect the ratio of IRS1 phosphorylation, so we examined Akt-mTOR signaling as another possible pathway. mTOR, a phosphorylation target of Akt, was stimulated and showed increased expression of amino acids [35]. mTOR is the catalytic subunit of the mTORC1 and mTORC2 complexes. If mTORC2 phosphorylation increases, Akt phosphorylation, and FoxO1 phosphorylation also increase, and this, in turn, increases POMC expression. On the other hand, mTORC1 activity causes a negative feedback loop in the insulin signaling pathway. Activation of mTORC1 leads to the phosphorylation of downstream S6K, which participates in several processes, including protein synthesis and proliferation $[15,36]$. Further, increased activity of the mTOR-S6K signaling pathway leads to serine phosphorylation of IRS1, creating a negative feedback loop in insulin signaling that attenuates insulin sensitivity $[37,38]$. In our study, we considered that YNCRG stimulated mTORC2 phosphorylation because phosphorylation of factors involved in signaling through mTORC1, such as S6K and IRS1, did not change in the two groups. We concluded that YNCRG induced appetite suppression by activation of the mTORC2-Akt FoxO1-POMC signaling pathway in the hypothalamus of SHRCP rats (Fig. 10).

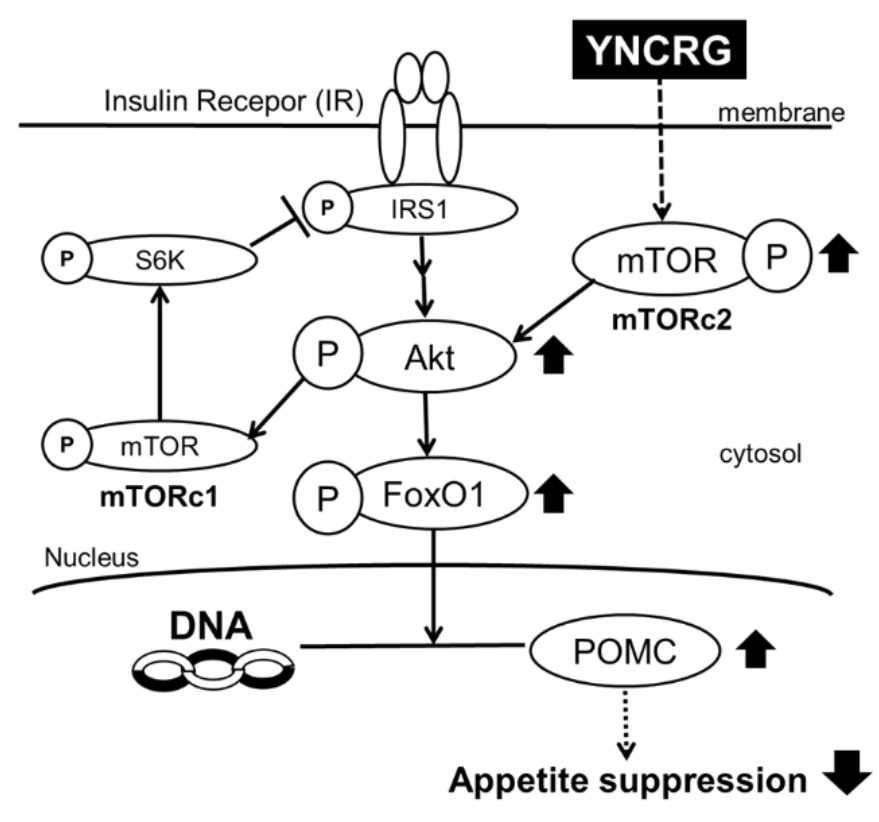

Figure 10 Signaling of appetite suppression in the hypothalamus of the YNCRG-treated rats. 
YNCRG treatment for eight weeks also led to body fat mass reduction. To understand the mechanism of this reduction, we investigated the expression levels of lipid metabolism-related proteins and genes in adipose tissue of SHRCP and WKY rats. LKB1/AMPK signaling is known to be involved in lipid metabolism [39]. In this study, AMPK, the main enzyme in the lipid metabolic pathway, and LKB1 were significantly phosphorylated in the YNCRG group. Moreover, PKA, an upstream factor of LKB1, was affected by YNCRG treatment, suggesting that YNCRG can promote lipid metabolism through the PKA-LKB1-AMPK pathway. A previous study reported that AMPK is also activated by an upstream factor, CaMKK [40]. The activation of AMPK in the YNCRG group did not affect the phosphorylation of CaMKK, indicating that activation of AMPK by YNCRG was not dependent on CaMKK. YNCRG also affected the lipogenesis-related protein ACC and lipolysis protein HSL but did not upregulate the level of $C / E B P \alpha, C / E B P \beta$, or PPAR $\gamma$. These results indicate that YNCRG inhibited ACC and promoted HSL expression, suggesting that YNCRG is involved in both lipogenesis inhibition and lipolysis promotion. We suggest that YNCRG may reduce body fat mass by activation of lipid metabolism, inhibition of lipogenesis, and promotion of lipolysis via AMPK activation in adipose tissue (Fig. 11).

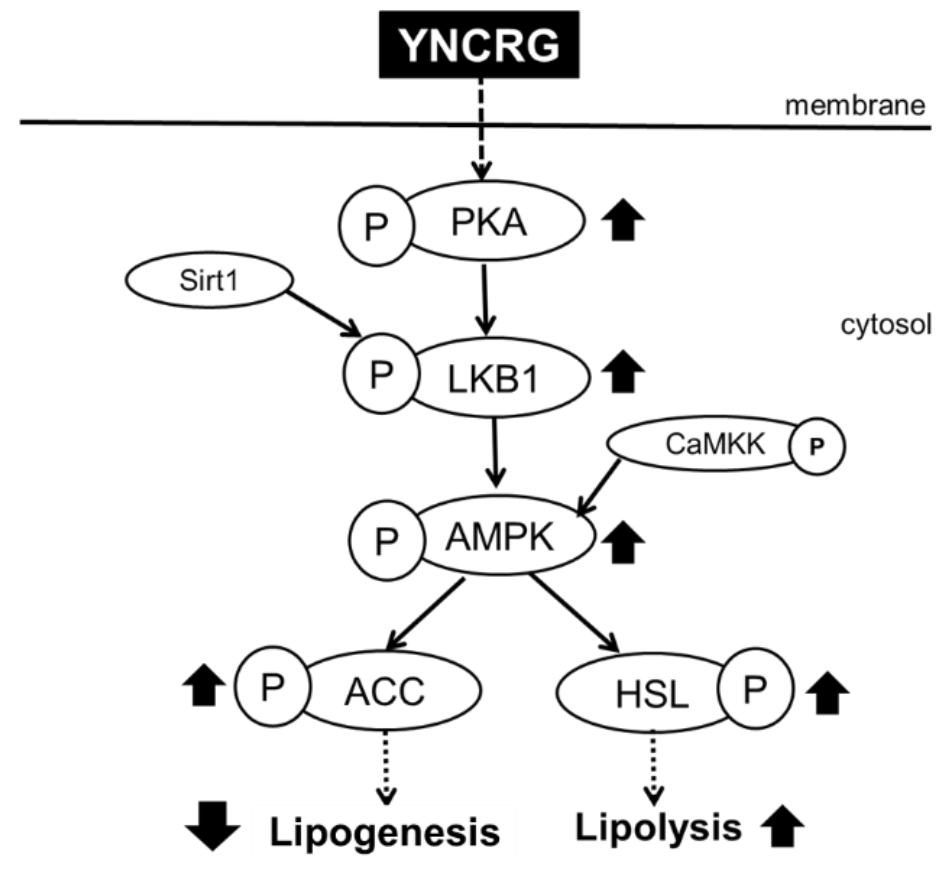

Figure 11 Signaling of fat mass reduction in the adipose tissue of YNCRG-treated rats.

YNCRG inhibited body weight gain. The mechanisms involved are appetite suppression in the hypothalamus and reduction in body fat mass due to improvement in the lipid metabolic pathway caused by lipogenesis inhibition and lipolysis promotion in adipose tissue in SHRCP rats.

YNCRG contains five extracts and chemical compounds from herbal plants. Previously, we reported that L-Cit reduced obesity via appetite suppression using high-fat diet-fed Sprague Dawley (SD) rats [11]. Furthermore, the regulation of POMC expression through GABA ${ }_{B}$ receptors is essential for proper energy balance [12]. YNCRG contains L-Cit and GABA, both of which are thought to be involved in body weight gain reduction effect via appetite suppression. Regarding other compounds, Eucommia ulmoides has demonstrated anti-hypertensive, anti-hyperlipidemic, 
and anti-obesity effects [41-45] and Monascus purpureus also showed an anti-obesity effect via the inhibition of differentiation and lipogenesis in high-fat diet-fed obese rats [6].

Also, some studies have demonstrated that the principal bioactive compounds from Morinda citrifolia have potential beneficial effects in obesity and obesity-related metabolic dysfunction [46]. It is suggested that components Eucommia ulmoides, Monascus purpureus, and Morinda citrifolia may be involved in a bodyweight gain suppressive effect due to body fat mass reduction via an improvement in the lipid metabolic pathway.

However, there are many unknown mechanisms regarding the detailed interactions of YNCRG, i.e., a mixture of five components, so we plan to investigate the detailed mechanism in future studies.

\section{Conclusions}

We elucidated the mechanism of action and possible synergistic effects of YNCRG on MetS through amelioration of appetite suppression and lipid metabolism in SHRCP rats.

In our study, AST and ALT levels, which indicate liver function, were not affected. Besides, YNCRG had no negative effect on bowel movements or activities. Moreover, we observed the dissected stomach and intestines, and could not find any abnormal features. Therefore, we consider that the YNCRG dose used in our study has no toxicity or side effects.

Further studies are planned. YNCRG may become a new treatment option for MetS, especially due to its inhibition of body weight gain due to body fat mass reduction via appetite suppression and improvement in the lipid metabolic pathway. It can be used in clinical practice in the future.

\section{Abbreviations}

MetS: metabolic syndrome

POMC: proopiomelanocortin

JAK2: janus kinase 2

STAT3: signal transducer and activator of transcription 3

IR: insulin receptor

IRS1: insulin receptor substrate 1

Akt: protein kinase $B$

FoxO1: forkhead box 01

AMPK: AMP-activated protein kinase

CaMKK: Calcium-calmodulin/dependent protein kinase

LKB1: liver kinase B1

PPAR $\gamma$ : peroxisome proliferator-activated receptor $\gamma$

C/EBP: CCAAT/enhancer-binding protein

SREBP-1c: sterol regulatory element binding protein $1 \mathrm{c}$

ACC: acetyl-CoA carboxylase

FAS: fatty acid synthase

Sirt1: Sirtuin 1

HSL: hormone-sensitive lipase

SHRCP: SHR.Cg-Lepr ${ }^{\mathrm{cP}} / \mathrm{NDmcr}$

L-Cit: L-Citrulline 
GABA: gamma-aminobutyric acid WKY: Wister Kyoto

OGTT: oral glucose tolerance test NFBG: non-fasting blood glucose

TC: total cholesterol

TG: triglyceride

AST: aspartate aminotransferase

ALT: alanine aminotransferase

ALB: albumin

BUN: blood urea nitrogen

Cre: creatinine

UA: uric acid

TP: total protein

UN: urine nitrogen

SBP: Systolic blood pressure

DBP: diastolic blood pressure

FBG: fasting blood glucose

AUC: area under the curve

BIA: bioelectrical impedance analysis

PVDF: polyvinylidene difluoride

mTOR: mammalian target of rapamycin

S6K: S6 kinase

PKA: protein kinase $A$

Agrp: agouti related protein

GAPDH: glyceraldehyde-3-phosphate dehydrogenase

Sirt1: sirtuin1

SD: Sprague Dawley

\section{Acknowledgments}

Not applicable.

\section{Author Contributions}

Author contributions are as follows: study design, MK, MH, TL and MG; experiments, MK, MH, $\mathrm{PT}$, DL and LW; data analysis, MK; providing the five plant materials, $\mathrm{LW}, \mathrm{ZH}, \mathrm{YZ}, \mathrm{TN}$ and $\mathrm{MN}$; and writing of the manuscript, MK, TL and MG.

\section{Funding}

Not applicable.

\section{Competing Interests}

The authors have declared that no competing interests exist. 


\section{References}

1. Wang $T$, Kusudo $T$, Takeuchi $T$, Yamashita $Y$, Kontani $Y$, Okamatsu $Y$, et al. Evodiamine inhibits insulin-stimulated mTOR-S6K activation and IRS1 serine phosphorylation in adipocytes and improves glucose tolerance in obese/diabetic mice. PLoS One. 2013; 8: e83264.

2. Kopelman PG. Obesity as a medical problem. Nature. 2000; 404: 635-643.

3. Susanti VY, Sasaki T, Yokota - Hashimoto H, Matsui S, Lee YS, Kikuchi O, et al. Sirt1 rescues the obesity induced by insulin-resistant constitutively-nuclear FoxO1 in POMC neurons of male mice. Obesity. 2014; 22: 2115-2119.

4. Park HK, Ahima RS. Leptin signaling. Prime Rep. 2014; 6: 73.

5. White MF. IRS proteins and the common path to diabetes. Am J Physiol Endocrinol Matab. 2002; 283: E413-E422.

6. Zoncu R, Efeyan A, Sabatini DM. mTOR: From growth signal integration to cancer, diabetes and ageing. Nat Rev Mol Cell Biol. 2011; 12: 21-35.

7. Kim JE, Chen J. Regulation of peroxisome proliferation-activated receptor-activity by mammalian target of rapamycin and amino acid in adipogenesis. Diabetes. 2004; 53: 27482756.

8. Liu H, Wang J, Liu M, Zhao H, Yaqoob S, Zheng M, et al. Antiobesity effects of ginsenoside Rg1 on 3T3-L1 preadipocytes and high fat diet-induced obese mice mediated by AMPK. Nutrients. 2018; 10: 830.

9. Kou R, Sartoretto J, Michel T. Regulation of Rac1 by simvastatin in endothelial cells: Differential roles of AMP-activated protein kinase and calmodulin-dependent kinase kinasebeta. J Biol Chem. 2009; 284: 14734-14743.

10. Hardie DG. The AMP-activated protein kinase pathway-new players upstream and downstream. J Cell Sci. 2004; 117: 5479-5487.

11. Mota de Sa P, Richard AJ, Hang H, Stephens JM. Transcriptional regulation of adipogenesis. Compr Physiol. 2017; 7: 635-674.

12. Smith U, Kahn BB. Adipose tissue regulates insulin sensitivity: Role of adipogenesis, de novo lipogenesis and novel lipids. J Intern Med. 2016; 280: 465-475.

13. Fujikawa T, Hirata T, Wada A, Kawamura N, Yamaguchi Y, Fujimura K, et al. Chronic administration of Eucommia leaf stimulates metabolic function of rats across several organs. Br J Nutr. 2010; 104: 1868-1877.

14. Zheng J, Liu D, Zhao SQ, Su J, Yan QP, Chen L, et al. Enzymatic extraction and antibacterial activity of aucubin from Eucommia ulmoides leaves. J Chinese Med Mater. 2012; 35: 304-306.

15. Hung MY, Fu TYC, Shih PH, Lee CP, Yen GC. Du-Zhong (Eucommia ulmoides Oliv.) leaves inhibits CCl4-induced hepatic damage in rats. Food Chem Toxicol. 2006; 44: 1424-1431.

16. Luo LF, Wu WH, Zhou YJ, Yan J, Yang GP, Ouyang DS. Antihypertensive effect of Eucommia ulmoides Oliv. Extracts in spontaneously hypertensive rats. J Ethnopharmacol. 2010; 129: 238-243.

17. Ma J, Li Y, Ye $Q$, Li J, Hua $Y$, Ju D, et al. Constituents of red yeast rice, a traditional Chinese food and medicine. J Agric Food Chem. 2000; 48: 5220-5225.

18. $\mathrm{Yu} \mathrm{HC}$, Hsu JL, Chang $\mathrm{Cl}$, Tan FJ. Antioxidant properties of porcine liver proteins hydrolyzed using Monascus purpureus. Food Sci Biotechnol. 2017; 26: 1217-1225. 
19. Lee CL, Wen JY, Hsu YW, Pan TM. Monascus-fermented yellow pigments monascin and ankaflavin showed anti-obesity effect via the suppression of differentiation and lipogenesis in obese rats fed a high-fat diet. J Agric Food Chem. 2013; 61: 1493-1500.

20. Whistler WA. Traditional and herbal medicine in the Cook Islands. J Ethnopharmacol. 1985; 13: 239-280

21. Wang MY, West BJ, Jensen CJ, Nowicki D, Su C, Palu AK, et al. Morinda citrifolia (Noni): A literature review and recent advances in Noni research. Acta Pharmacol Sin. 2002; 23: 11271141

22. Singh YN, Ikahihifo T, Panuve M, Slatter C. Folk medicine in Tonga. A study of the use of herbal medicines for obstetric and gynaecological conditions and disorders. J Ethnopharmacol. 1984; 12: 305-329

23. Solomon IN. Tahitian Noni Juice: The tropical fruit with 101 medical uses. Salt Lake City: Woodland Publishing; 1999.

24. Rimando A. Perkins-Veazie P. Determination of citrulline in watermelon rind. J Chromatogr A. 2005; 1078: 196-200.

25. Kudo,M, Yoshitomi H, Momoo M, Suguro S, Yamagishi Y, Gao M. Evaluation of the effects and mechanism of L-citrulline on anti-obesity by appetite suppression in obese/diabetic KK-Ay mice and high-fat diet fed SD rats. Biol Pharm Bull. 2017; 40: 524-530.

26. Ito $Y$, Banno R, Shibata M, Adachi K, Hagimoto S, Hagiwara D. GABA type B receptor signaling in proopiomelanocortin neurons protects against obesity, insulin resistance, and hypothalamic inflammation in male mice on a high-fat diet. J Neurosci. 2013; 33: 1716617173.

27. Kawai K, Sakairi T, Harada S, Shinozuka J, Ide M, Sato H, et al. Diet modification and its influence on metabolic and related pathological alterations in the SHR/NDcmcr-cp rat, an animal model of the metabolic syndrome. Exp Toxicol Pathol. 2012; 64: 333-338.

28. Nagase M, Yoshida S, Shibata S, Nagase T, Gotoda T, Ando K, et al. Enhanced aldosterone signaling in the early nephropathy of rats with metabolic syndrome: Possible contribution of fat-derived factors. J Am Soc Nephrol. 2006; 17: 3438-3446.

29. Li L, Yoshitomi H, Wei Y, Qin L, Zhou J, Xu T, et al. Tang-Nai-Kang alleviates pre-diabetes and metabolic disorders and induces a gene expression switch toward fatty acid oxidation in SHR. Cg-Leprcp/NDmcr Rats. PLoS One. 2015; 10: e0122024.

30. Smith DL, Johnson M, Nagy T. Precision and accuracy of bioimpedance spectroscopy for determination of in vivo body composition in rats. Int J Body Compos Res. 2009; 7: 21-26.

31. Liu Q, Li W, Nagata K, Fu H, Okada S, Tanabe I, et al. Isolation, structural elucidation, and liquid chromatography-massspectrometry analysis of steroidal glycosides from Polygonatum odoratum. J Agric Food Chem. 2018; 66: 521-531.

32. Morrison CD, Morton GJ, Niswender KD, Gelling RW, Schwartz MW. Leptin inhibits hypothalamic Npy and Agrp gene expression via a mechanism that requires phosphatidylinositol 3-OH-kinase signaling. Am J Physiol Endocrinol Metab. 2005; 289: 10511057.

33. Banks AS, Davis SM, Bates SH, Myers MG. Activation of downstream signals by the long form of the leptin receptor. J Biol Chem. 2000; 275: 14563-14572.

34. Tartaglia L. The leptin receptor. J Biol Chem. 1997; 272: 6093-6096. 
35. Tremblay F. Matette A. Amino acids and insulin signaling via the mTOR/p70S6 kinase pathway: A negative feedback mechanism leading to insulin resistance in skeletal muscle cells. J Biol Chem. 2001; 276: 38052-38060.

36. Shima $H$, Pende $M$, Chen $Y$, Fumagalli $S$, Thomas $G$, Kozma SC. Disruption of the p70(s6k)/p85(s6k) gene reveals a small mouse phenotype and a new functional S6 kinase. EMBO J. 1998; 17: 6649-6659.

37. Um SH, Frigerio F, Watanabe $M$, Picard $F$, Joaquin $M$, Sticker $M$, et al. Absence of S6K1 protects against age-and diet-induced obesity while enhancing insulin sensitivity. Nature. 2004; 431: 200-205.

38. Um SH, D'Alessio D, Thomas G. Nutrient overload, insulin resistance, and ribosomal protein $\mathrm{S} 6$ kinase 1, S6K1. Cell Metab. 2006; 3: 393-402.

39. Mirouse V, Billaud M. The LKB1/AMPK polarity pathway. FEBS Lett. 2011; 585: 981-985.

40. Chen $Q$, Liu M, Yu H, Li J, Wang S, Zhang $Y$, et al. Scutellaria baicalensis regulates FFA metabolism to ameliorate NAFLD through the AMPK-mediated SREBP signaling pathway. J Nat Med. 2018; 72: 655-666.

41. Medica CM. Jiangsu New Medical College. Shanghai: Shanghai Science and Technology Publishing House; 1977.

42. Fujikawa T, Hirata T, Wada A, Kawamura N, Yamaguchi Y, Fujimura K, et al. Chronic administration of Eucommia leaf stimulates metabolic function of rats across several organs. Br J Nutr. 2010; 104: 1868-1877.

43. Namba T. Studies on Tu-Chung leaves (I), Pharmacological effects of the water extract in vivo. J Trad Med. 1986; 3: 89-97.

44. Metori K, Ohashi S, Takahashi S, Tamura T. Effects of du-zhong leaf extract on serum and hepatic lipids in rats fed a high-fat diet. Biol Pharm Bull. 1994; 17: 917-920.

45. Hosoo S, Koyama M, Watanabe A, Ishida R, Hirata T, Yamaguchi Y, et al. Preventive effect of Eucommia leaf extract on aortic media hypertrophy in Wistar-Kyoto rats fed a high-fat diet. Hypertens Res. 40; 2017: 546-551.

46. Inada AC, Figueiredo PS, Santos-Eichler RAD, Freitas KDC, Hiane PA, Castro APD, et al. Morinda citrifolia Linn. (Noni) and its potential in obesity-related metabolic dysfunction. Nutrients. 2017; 9: 540. 
OBM Integrative and Complementary Medicine 2020; 5(3), doi:10.21926/obm.icm.2003033

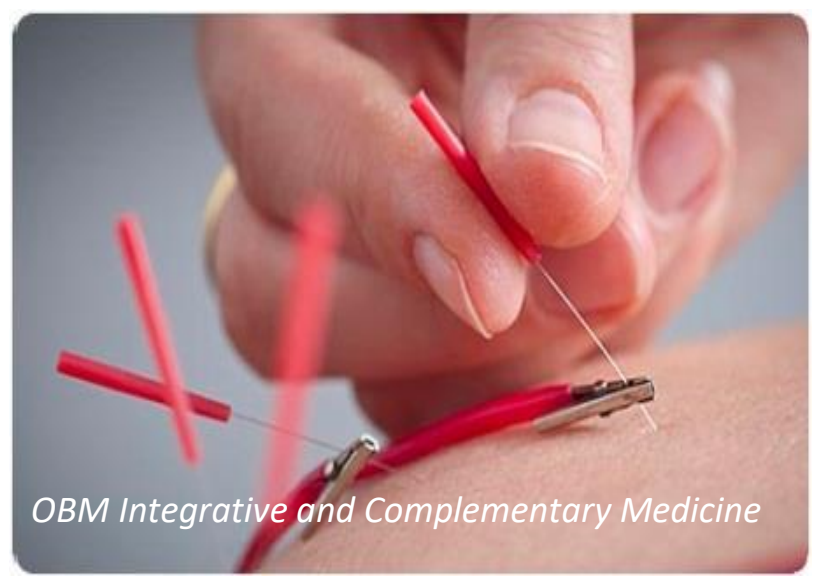

Enjoy OBM Integrative and Complementary Medicine by:

1. Submitting a manuscript

2. Joining in volunteer reviewer bank

3. Joining Editorial Board

4. Guest editing a special issue

For more details, please visit:

http://www.lidsen.com/journals/icm 